\title{
Civilisations
}

Revue internationale d'anthropologie et de sciences

humaines

56 | 2007

Après la catastrophe

\section{De l'exil à l'asile : témoignages et authenticité culturelle des candidats tibétains au statut de réfugié politique en Belgique}

\author{
Isabelle Henrion-Dourcy
}

\section{OpenEdition}

Journals

Édition électronique

URL : http://journals.openedition.org/civilisations/157

DOI : $10.4000 /$ civilisations. 157

ISSN : 2032-0442

\section{Éditeur}

Institut de sociologie de l'Université Libre de Bruxelles

\section{Édition imprimée}

Date de publication : 1 décembre 2007

Pagination : 121-158

ISBN : 2-87263-016-3

ISSN : 0009-8140

\section{Référence électronique}

Isabelle Henrion-Dourcy, « De l'exil à l'asile : témoignages et authenticité culturelle des candidats tibétains au statut de réfugié politique en Belgique », Civilisations [En ligne], 56 | 2007, mis en ligne le 01 décembre 2010, consulté le 19 avril 2019. URL : http://journals.openedition.org/civilisations/157 ; DOI : 10.4000/civilisations. 157 


\title{
De l'exil à l'asile \\ Témoignages et authenticité culturelle des candidats tibétains au statut de réfugié politique en Belgique ${ }^{1}$
}

\author{
Isabelle HENRION-DOURCY
}

\begin{abstract}
Résumé : Cet article consiste en une analyse des témoignages livrés par des réfugiés tibétains lors la procédure d'asile politique qu'ils ont engagée en Belgique. Il est fondé sur une enquête ethnographique de quinze mois et situe les informations recueillies dans le contexte historique, politique et social des différentes trajectoires de migration des candidats. Leur situation se révèle particulière en raison de l'absence d'un pays souverain. Le contenu de ces récits est examiné, ainsi que leur mode d'élaboration : examinateurs belges et candidats tibétains ont dû traverser une longue période d'ajustement culturel, la rhétorique du témoignage politique étant un mode discursif inconnu au Tibet traditionnel, et le régime de la vérité - ou de la réalité de l'expériencerecouvrant des notions différentes de part et d'autre. Aux yeux des candidats eux-mêmes, plus importantes sont les implications idéologiques de ces récits : ceux-ci trahissent le plus souvent l'origine de leurs auteurs, qui sont soit nés au Tibet (acceptables pour l'asile politique) soit nés en exil en Inde ou au Népal (alors rejetés, car considérés comme non soumis à des violations des droits de l'homme). Appuyant sur cette fracture sensible entre Tibétains, l'audition au Commissariat Général aux Réfugiés et aux Apatrides, examen de nature juridique destiné à juger les actions d'un individu et les risques de persécution qu'il encourt, s'est transformée en une évaluation de leur origine géographique et, partant, de leur authenticité culturelle - une authenticité dont chaque groupe se déclare pourtant le parfait détenteur.
\end{abstract}

Mots-clés : réfugiés, asile politique, récit, authenticité culturelle, Tibet.

Summary: This article examines the narratives given by Tibetan refugees in the course of their application for political asylum in Belgium. Based on fifteen months of ethnographic fieldwork, it provides a broad historical, political and social contextualization of their different migration trajectories. The situation of the Tibetan asylum seekers is inflected by the fact that they do not have a sovereign country. The content of their narratives is examined, as well as the modalities of their construction: Belgian interviewers and Tibetan refugees have had to adjust culturally, the rhetoric of political testimony being unknown in traditional Tibet, and the regime of truth - or of what constitutes the reality of experience - being constructed differently on both parts. Yet, the crucial concern of the Tibetans themselves is the ideological implications of these narratives: they most often give away the origin of their authors, who are either born in Tibet (thus qualifying for asylum) or born in exile in India or Nepal (thus rejected because hailing from a country considered free of human rights violations). While pressing on this sensitive fracture between groups of Tibetans, the official audition at the Commissariat has gone from being a legal examination of the deeds of an individual and his risks of persecution, to being an evaluation of the geographic origin of the candidates, hence of their cultural authenticity - the very notion of which each group claims to be the most adequate representative.

Key words: refugies, political asylum, narrative, cultural authenticity, Tibet.

1 Que soient ici remerciés les Tibétains résidant en Belgique qui ont accepté de dévoiler pour moi un petit morceau de leur « intimité culturelle », ainsi que Dominique De Mees (Amnesty International) et Peggy Baten (CGRA), dont la longue expérience et le généreux partage d'informations ont nourri les réflexions de cet article. Les noms des Tibétains sont tous des pseudonymes. 
Les Chinois s'établissent en masse au Tibet.

Les Tibétains fuient en Inde.

Les Tibétains d'Inde émigrent en Occident.

Et les Occidentaux vont faire des affaires... en Chine.

C'est ça, en fin de compte, la modernité?

Quelle est cette insatisfaction qui pousse ainsi chacun à partir de chez lui?

(Yonten, réfugié en Belgique)

P ar quel terme désigne-t-on un yak femelle de deux ans ? » La question de l'agent traitant du Commissariat Général aux Réfugiés et aux Apatrides (CGRA) laisse Tsering pantois. Il ne s'attendait pas à ce type de questions. «Euh... dri », ose-t-il ce n'est que le terme générique employé pour ces bovidés de sexe féminin. C'est sa deuxième audition au Commissariat. Il est arrivé en Belgique il y a trois ans. Sa demande d'asile a été rejetée lors de la première audition en recevabilité à l'Office des Etrangers. Cette deuxième comparution au CGRA, destinée à l'interroger en profondeur, est donc décisive. Cette fois, il s'est bien préparé. Il a révisé les points importants de son récit, surtout les chiffres et les noms : sa date de naissance, les dates de ses activités politiques, de son incarcération, le nom de ses complices, la description de la prison, les détails de son évasion et de sa fuite au Népal, le nom des villages traversés de nuit, celui du passeur, celui de son contact à Katmandou, son arrivée en Belgique. Il est arrivé tôt le matin au Commissariat, un peu nerveux, mais il s'est petit à petit détendu en faisant le récit de ces événements marquants. L'interrogateur écoute patiemment la traduction qu'en fait son interprète, prend des notes, le relance par une question inattendue. Mais quel rapport cette question a-t-elle donc avec son récit, avec lui en tant qu'individu, avec sa crédibilité en tant que réfugié politique ? Comment Tsering pouvait-il anticiper que ce serait sur ce type de questions générales sur la vie quotidienne au Tibet qu'allait se jouer une partie cruciale de sa vie, à savoir l'obtention du statut de réfugié politique et des droits sociaux qui lui sont assortis?

L'étude qui suit est fondée sur un travail ponctuel d'accompagnement, entre septembre 2003 et décembre 2004, d'une quarantaine de Tibétains requérant l'asile politique en Belgique. Je me propose d'analyser les témoignages qu'ils ont livrés dans le cadre de la procédure d'asile, en examinant à la fois leur contenu, leur mode d'élaboration et leurs implications. A partir de ces récits, d'entretiens informels et d'une mise en perspective historique et sociale des migrations tibétaines, je tâcherai de jeter un éclairage sur l'expérience de ces jeunes réfugiés tibétains en Occident. La présence de réfugiés tibétains en Belgique pourrait paraître marginale : ils sont à peine plus d'un millier. Pourtant, cette immigration est remarquable. D'abord en raison de la population souche, constituée de moins de cent cinquante mille exilés en Asie du Sud. Ensuite, parce qu'il s'agit d'une immigration en Occident particulièrement récente. En tant que "phénomène de masse » (à l'échelle tibétaine), elle a moins de quinze ans. En ce qui concerne la Belgique, la quasi-totalité des réfugiés sont arrivés après 2002. Autres faits saillants : ils sont tous «politisés » (du moins ils ont tous fait une demande d'asile politique), mais comme ils n'ont pas de pays souverain, et qu'ils sont presque tous inexpulsables vers la Chine, leur procédure d'asile est un peu plus complexe que pour d'autres nationalités. Enfin, il faut 
souligner qu'au sein de l'Europe, c'est la Belgique qui a accueilli le plus grand nombre de Tibétains ces dernières années. On peut donc parler d'un mini-boom d'immigration tibétaine en Belgique. Ces néo-réfugiés ne correspondent pas, en majorité, aux images désolantes véhiculées fréquemment par les médias : venus en avion et munis de (faux ou vrais) passeports, quasiment invisibles au sein de leur société d'accueil (numériquement et pénalement), ils pourraient à première vue apparaître comme des « réfugiés de luxe » par rapport à ces « misérables mers d'humanité » (Malkki 1996 : 377) en Afrique centrale, à ces naufragés d'embarcations de fortune en Méditerranée, ou encore à ces familles qui fuient clandestinement l'Asie centrale à la recherche d'un « Dernier caravansérail »². Et pourtant, l'arrivée en Occident de ces réfugiés tibétains constitue aussi, pour chacun d'entre eux, un parcours semé d'embûches et d'incompréhensions, une dernière étape d'un très long parcours, souvent marqué simultanément par le tragique et par l'absurde.

Certes, les témoignages dont il va être ici question sont formels, produits dans et pour le contexte d'une procédure bureaucratique, mais ils n'en sont pas moins de riches indicateurs des enjeux actuels auxquels cette communauté de réfugiés est confrontée. Ces récits sont censés être des témoignages autobiographiques où les narrateurs doivent produire la preuve d'une persécution individuelle. En réalité, ils relayent de grands stéréotypes véhiculés officiellement dans les communautés tibétaines d'origine. A travers ces voix individuelles, ce sont donc avant tout des grands lieux communs qui sont donnés à entendre: sur l'histoire et sur la nation tibétaine, préoccupations prioritaires d'une diaspora jeune à forte cohésion culturelle. Ces lieux communs sont à chaque fois intégrés, refaçonnés et réactualisés à travers le filtre d'expériences singulières. Dans ce processus de captage personnel de représentations collectives, l'origine géographique et les trajectoires des migrants prennent une importance considérable. Les réfugiés tibétains proviennent tous d'Inde et du Népal, pays qui constituent en quelque sorte la plaque tournante de l'émigration tibétaine, mais ils n'arrivent pas pour autant égaux en Occident.

Le problème crucial soulevé par la procédure d'asile n'est pas, pour les candidats tibétains, l'énorme difficulté de compréhension interculturelle entre les examinateurs belges et eux-mêmes - un ajustement qui a nécessité de longs mois de dialogue. De manière plus fondamentale, l'enjeu est idéologique. Car, ce que la procédure d'asile a mis en lumière, c'est la fracture qui divise dorénavant le peuple tibétain : le fossé qui sépare, plus particulièrement chez les jeunes, ceux qui sont nés au Tibet de ceux nés en exil en Inde ou au Népal. C'est dans cette fracture irréconciliable que réside la conséquence la plus tragique, la plus profonde aussi, de « la catastrophe ». C'est également la faille la moins avouable d'une diaspora qui affiche son unité comme instrument politique. C'est encore l'angle mort de la plupart des travaux académiques sur les Tibétains (sauf Yeh 2002, 2007). Pour les instances d'accueil belges, les Tibétains du Tibet (qui ont en réalité longuement transité par l'Inde ou le Népal) peuvent remplir les conditions de l'asile politique, mais il n'en va pas de même pour les réfugiés nés dans ces deux derniers pays. Or, de très nombreux requérants sont nés en exil. Ils prétendent provenir du Tibet, mais ils n'en ont jamais foulé le sol. Ils n'ont jamais été confrontés directement à l'oppression politique qu'ils dénoncent. Leur bagage culturel, leurs représentations identitaires et leur

2 Voir l'article de Françoise Lauwaert dans ce volume, consacré au spectacle éponyme du Théâtre du Soleil, réalisé à partir des récits de tels réfugiés (iraniens, afghans, kurdes, russes, etc.) recueillis dans des camps en France et en Australie. 
vécu social sont très différents de ceux des Tibétains du Tibet. Mais comment différencier les uns des autres puisqu'ils parlent la même langue ? Les candidats ont donc dû subir le crible de questions portant sur leur mode de vie (paysan, pastoral ou urbain) afin de distinguer les « vrais » Tibétains des autres. Il y aurait donc des Tibétains plus vrais que d'autres, alors que les uns comme les autres, ou plus exactement les uns par opposition aux autres, et avec une charge émotive tout aussi importante, se considèrent comme les détenteurs légitimes de l'identité culturelle de leur peuple.

Ainsi, l'audition au CGRA, examen de nature juridique reposant sur le régime de la preuve, destiné à juger les actions passées d'un individu et les risques de persécution qu'il encourt à l'avenir, s'est-elle progressivement transformée en un examen d'un autre type : une évaluation, par une instance officielle à la fois étrange et étrangère aux yeux des Tibétains, de leur origine géographique et, partant, de leur authenticité culturelle. Leurs témoignages, loin de n'être que des dossiers juridiques ou des porte-voix d'une cause politique, sont devenus des zones de fracture : une fracture collective (entre groupes de Tibétains en Belgique) tout autant que personnelle (l'échec en procédure d'asile vécu comme impuissance personnelle à s'inscrire dans une tibétanité reconnue) ${ }^{3}$.

Avant d'aborder ces récits, prenons toutefois le temps de situer cette néo-migration dans son contexte historique et idéologique.

\title{
Le contexte d'origine : Tibet occupé et diaspora en Asie du Sud
}

\author{
Le vent disperse dans les dix directions \\ Les pousses issues d'une même tige. \\ De la même, manière, le karma sépare \\ Les hommes nés ensemble ${ }^{4}$.
}

Pour le meilleur comme pour le pire 5 , la « catastrophe » tibétaine à laquelle répondent les considérations de cet article a rencontré dans les médias occidentaux un écho suffisamment important pour qu'il suffise d'en rappeler brièvement les faits majeurs ${ }^{6}$. Même si cet écho est partiel ou partial, il faut reconnaître que les Tibétains semblent avoir bénéficié, plus que d'autres, d'une attention internationale qui se prodigue avec de grandes disparités. L'aura médiatique du Dalai Lama, lauréat du prix Nobel de la paix en 1989, a

3 Soulignons que depuis 2004, moment où s'arrêtent les observations sur lesquelles sont basées cet article, plusieurs paramètres de la situation étudiée ont connu des modifications : la procédure d'asile a été réformée (2006), les examinateurs belges et les candidats tibétains ont développé une meilleure compréhension mutuelle (le contenu de l'audition a donc quelque peu changé) et enfin le profil des candidats s'est modifié, puisque rares sont à présent ceux que l'on peut identifier comme provenant de l'exil (ils sont presque tous originaires du Tibet).

4 sDong-po gcig las skyes-pa yi / rtsa-rnams rlung-gis phyogs-bcur 'thor / de-bzhin lhan-cig skyes-pa yi / skye-bo las kyis so-sor 'bral. Tiré de la célèbre anthologie des sentences de Sakya Pandita Kunga Sonam Gyaltsen (1182-1251).

5 Barnett (2001) démontre que les diverses formes d'activisme en Occident ont tout autant aidé la cause politique tibétaine qu'elles lui ont nui.

6 On se reportera à Shakya (1999), Sperling (2004), Buffetrille et Ramble (1998) et Buffetrille et Blondeau (2002) pour une analyse rigoureuse des relations sino-tibétaines avant et depuis 1950. 
certainement contribué à cette popularité. Mais il faut aussi y ajouter la fascination qu'a entretenue de longue date l'Occident pour la pratique religieuse et l'environnement naturel de l'Himalaya, laquelle a lentement façonné l'image du Tibet comme un exotique paradis terrestre, corrompu depuis peu par l'expression la plus haïssable de la modernité.

Depuis la mainmise de la République populaire de Chine en 1951, les Tibétains ont été confrontés à ce qui constitue en réalité une série de bouleversements, imposés dans la violence et vécus comme autant de «catastrophes » d'intensité toujours plus grande. La première période est la plus brutale : annexion militaire, exactions dans les provinces orientales puis centrales qui conduisent au soulèvement de Lhasa en 1959, répression de l'insurrection?', exil du Dalai Lama en Inde suivi par près de cent mille des siens. Dorénavant arrimés au devenir de la Chine dans son ensemble, conceptuellement déclassés au rang de minorité ethnique, et surtout issus d'une société traditionaliste qui ne les a en rien préparés à un avenir révolutionnaire, les Tibétains doivent rapidement s'habituer à un appareil d'Etat particulièrement puissant, à des politiques décidées dans un autre univers culturel - à Pékin -, et à la cadence soutenue des bouleversements qui affectent uniformément la Chine : réformes socialistes, purge de l'élite religieuse et aristocratique, emprisonnement et travaux forcés pour les opposants, et enfin, point culminant, Révolution culturelle (1966-1976). Les destructions sont colossales ; le sentiment de perte, abyssal. Le gouvernement tibétain en exil avance, sur la base d'un recensement par villages, le chiffre, invérifiable, d'un million deux cent mille morts (un Tibétain sur cinq) pour cette première période ${ }^{8}$. La quasi-totalité des monastères et une grande partie du patrimoine culturel (artéfacts, textes, même parures et objets personnels) ont été réduits en cendres.

La deuxième période, celle des années quatre-vingt, est celle de l'assouplissement politique général en Chine, qui s'est traduit au Tibet par une reconstruction sélective, à tous niveaux, des traditions culturelles les plus importantes : les monastères et les diverses formes de pratique religieuse, les arts populaires, l'enseignement et la promotion de la langue tibétaine. Cette revitalisation devient affirmation culturelle. Elle ravive les griefs d'une population mécontente : les manifestations indépendantistes de Lhasa entre 1987 et 1989 sont durement réprimées, la loi martiale est imposée jusqu'en 1991.

Le gouvernement central décide alors d'employer des moyens à la fois plus subtils et plus puissants pour susciter l'adhésion des Tibétains, comme il le fait dans les autres régions périphériques peuplées de minorités ethniques : c'est la troisième période, celle du développement économique, censé rendre inutile l'attachement aux valeurs traditionnelles et favoriser l'assimilation nationale. C'est aussi celle de l'intensification du transfert démographique depuis les plaines centrales, des projets d'infrastructure gigantesques, de l'endiguement de l'utilisation de la langue, du contrôle de la pratique religieuse et de l'interdiction de toute marque de sympathie pour le Dalai Lama (photographies, lecture ou écoute de ses discours). La liaison ferroviaire Pékin-Lhasa, achevée en 2006, acheminant près de deux millions de voyageurs supplémentaires par an, est brandie officiellement comme le symbole de ce nouveau Tibet : ouvert, prospère, touristiquement moderne. A la

7 Un document de l'Armée populaire de libération saisi en 1960 dénombre quatre-vingt sept mille victimes pour le seul mois qui a suivi la répression de l'insurrection.

8 http://www.tibet.com/WhitePaper/white5.html (consulté le 30 janvier 2007). 
marginalisation démographique, économique, linguistique et culturelle des Tibétains, persistante malgré un processus rapide de sinisation, répond leur aliénation quasi totale vis-à-vis de la population chinoise. A côté d'une minorité de privilégiés qui s'enrichit, une majorité s'enlise dans le désespoir. Tous s'interrogent sur la survie de leur langue, de leur culture et de leur mode de vie à mesure que s'intensifie l'exode rural.

\section{Changement de décor}

De l'autre côté de la barrière himalayenne, les cent mille Tibétains qui ont fui le pays en 1959 ont constitué, principalement en Inde, mais aussi au Népal (environ quinze mille) et au Bhoutan (mille cinq cents), une communauté en exil relativement bien coordonnée par une administration centrale (CTA, Central Tibetan Administration) sous la houlette du Dalai Lama9 . Les frontières de la Chine étant totalement hermétiques pendant les vingt premières années, la société de l'exil s'est reconstituée et développée sans aucun contact avec les six millions de compatriotes restés au pays. Tolérés avec largesse par l'Inde, économiquement dépendants de l'aide internationale, les Tibétains se sont établis dans des bourgades himalayennes (dont Dharamsala, leur chef-lieu) ou dans des villes (Delhi, Katmandou), la plupart d'entre eux étant néanmoins dirigés par le gouvernement de Delhi vers des camps agricoles dans le Sud de l'Inde. Comme toutes les diasporas (Malkki 1992, 1995), la diaspora tibétaine s'est fondée idéologiquement sur la réinterprétation de son histoire sur un mode mythique (en idéalisant la société telle qu'elle était avant le départ) et en s'attribuant une mission morale (préserver une culture " authentique », éradiquée du Tibet lui-même, pour pouvoir la retransmettre aux compatriotes lors d'un retour toujours espéré et sans cesse ajourné). Tout le projet de l'exil se résume donc à un conservatoire culturel, jugeant tout élément non traditionnel comme une trahison politique. La vie quotidienne, quant à elle, est rythmée par un calendrier patriotique (chant de l'hymne national à l'école, commémoration annuelle des rébellions, célébration du prix Nobel du Dalai Lama, rallies et manifestations dans les villes). Le combat pour le rétablissement de la souveraineté tibétaine sur le territoire est de ce fait une réalité profondément ancrée dans l'esprit des réfugiés.

La diaspora tibétaine est souvent perçue de l'extérieur comme une success story. C'est probablement dû à plusieurs facteurs : à l'image que ces Tibétains ont voulu donner d'eux-mêmes lorsqu'ils se sont ouverts à l'Occident à la fin des années soixante-dix, à une organisation interne relativement efficace et construite sur le modèle démocratique ${ }^{10}$, à un système éducatif (en langue tibétaine) très intégrateur, à un degré remarquable de préservation culturelle, et enfin à une cohésion sociale très forte de ses membres (endogamie relative, par exemple). Mais cette diaspora n'est pas uniforme. Elle comprend une grande proportion de réfugiés très pauvres et elle est parcourue de tensions importantes : entre courants réformistes et traditionalistes, entre acceptation et refus de l'acculturation

9 Nowak (1984) et Ginet (2000) dressent un bilan général de la situation des réfugiés tibétains en Inde et au Népal, de leur histoire récente, adaptation et problèmes. Voir aussi Assayag (1989) pour une étude ponctuelle dans un camp de réfugiés du Sud de l'Inde. 
indienne, entre nécessité de construire une identité pan-tibétaine et persistance des clivages prémodernes (régionaux et religieux). Elle connaît des heurts, enfin, entre tenants de la non-violence prônée par le Dalai Lama et défenseurs d'une attitude plus radicale. A ces tensions vient s'ajouter un sentiment de désespoir face à l'enlisement de la cause tibétaine, et de blocage face à l'impossibilité de s'intégrer pleinement dans l'économie indienne, voire internationale, en raison d'un statut juridique précaire ${ }^{11}$.

\section{Nouvelles vagues de migration}

Une nouvelle vague migratoire va profondément modifier la polarisation de ces deux contextes de vie. Avec la libéralisation politique mise en œuvre par Deng Xiaoping, les frontières s'entrouvrent : des Tibétains de l'exil peuvent venir rendre visite aux membres de leur famille. Des Tibétains du Tibet sont autorisés à se rendre en pèlerinage en Inde et au Népal. Peu d'exilés restent au Tibet, mais un grand nombre de Tibétains du Tibet s'établissent en Inde. Le durcissement politique qui fait suite aux manifestations indépendantistes amplifie l'exode. En réponse à cet afflux, des centres d'accueil sont créés à Katmandou et à Dharamsala. Petit à petit, les autorités indiennes et népalaises, et même l'administration centrale tibétaine, tentent d'endiguer le flot en promulguant des règles restrictives.

Cet exode s'est un peu ralenti, mais à l'heure actuelle, ce sont encore entre deux et trois mille fugitifs ${ }^{12}$ qui passent chaque année la frontière illégalement ${ }^{13}$. Ils entament ainsi à pied un périple de deux à six semaines à travers les montagnes ${ }^{14}$, sous la conduite

11 Le statut des Tibétains d'Inde est juridiquement délicat, et il l'est encore davantage au Népal (où ils ne seraient pas à l'abri d'une expulsion immédiate, si d'aventure le gouvernement venait à en décider ainsi), car aucun des deux pays n'a ratifié la Convention de Genève sur les réfugiés. Les Tibétains ne sont pas officiellement reconnus comme « réfugiés », mais simplement tolérés sur le territoire. En Inde, par exemple, beaucoup d'entre eux disposent d'un « RC » (registration certificate) renouvelable tous les ans, document essentiel dont le renouvellement est souvent source de tensions (Hess $2006: 82$ ). Suivant le mot d'ordre édicté par le gouvernement en exil, très rares sont les Tibétains qui ont opté pour la nationalité indienne ou népalaise (les grands commerçants transnationaux, principalement) : ce serait un aveu d'impuissance à faire reconnaître l'existence du Tibet comme pays. Ils restent donc, dans une très grande majorité, apatrides, et doivent entamer de longues procédures pour obtenir le « IC » (identity certificate), document qui leur permet de quitter le territoire indien et d'entrer dans les pays qui le reconnaissent comme l'équivalent d'un passeport (la plupart des pays... sauf la Chine, bien sûr). Soulignons enfin que les Tibétains ne sont pas les réfugiés les plus nombreux en Inde. En 2000, il y avait environ cent quarante-quatre mille Sri Lankais, cinquante-deux mille Birmans, quinze mille Népali du Bhoutan, vingt mille Bangladeshis et douze mille Afghans (Hess 2006 : note 4, p. 99).

12 Chiffres du Haut Commissariat aux Réfugiés (HCR) de l'ONU à Katmandou, depuis une dizaine d'années.

13 L'obtention d'un passeport, et surtout l'autorisation de quitter le pays, sont des démarches politicoadministratives rédhibitoires pour des personnes sans argent ni relations. Certains voyagent avec un passeport et dans le confort des transports routiers, mais l'immense majorité d'entre eux sont des fugitifs, à pied et sans papiers.

14 Voir les documentaires «Escape from Tibet» (Yorkshire Television 1994) et «Escape across the Himalayas » (ZDF 2000) qui ont accompagné de telles équipées. 
d'un passeur ${ }^{15}$, l'hiver de préférence (lorsque le zèle des garde-frontières est moindre ${ }^{16}$ ), multipliant de ce fait les risques de gelures et donc d'amputation à l'arrivée. Et ce n'est pas encore tout : suite à la pression exercée par la Chine, ils sont régulièrement remis par la police népalaise aux gardes chinois, quand ils ne sont pas rançonnés ou violentés par les villageois népalais.

Pourquoi prendre de tels risques ? Environ un tiers d'entre eux sont des religieux à la recherche d'un enseignement avancé, ou d'une pratique non entravée par un encadrement politique. Un autre tiers sont des enfants et des adolescents (aujourd'hui, provenant de zones plutôt rurales) qui souhaitent suivre une éducation en tibétain (et en anglais) inaccessible chez eux (Houston 2003 : 224). La plupart veulent simplement se trouver en présence du Dalai Lama. Les monastères et les écoles sont les seules structures qui peuvent accueillir sur le long terme les nouveaux venus. Les migrants qui ne sont ni religieux, ni en âge scolaire, sont encouragés à repartir au Tibet après un $a^{17}$. Beaucoup restent plus longtemps. Ils constituent officiellement $10 \%$ de la population de la diaspora en Inde ${ }^{18}$, résidant principalement à proximité de Dharamsala.

S'ils ont fui dans des conditions éprouvantes, ces " nouveaux venus » (sanjor [wa] en tibétain, terme qui en est venu à relever du registre de l'insulte) ne sont pas, pour autant, au bout de leurs peines. Alors que les tout premiers venus dans les années 1980 étaient accueillis à bras ouverts, comme le sont toujours les défecteurs de haut niveau, la cohabitation avec les réfugiés de longue date est devenue de plus en plus tendue. Au départ, il faut sans doute y voir, de la part des exilés de longue date, le choc, mêlé de dégoût, de découvrir des compatriotes bien moins «purs » et « pacifiques » que l'image qu'ils avaient construite d'eux. Qualifiés de «bruts », ou « crus » (kacha, en hindi), ou de « cerveaux pas mûrs » (leba jangu en tibétain), ils ont surpris par leur parler dialectal éloigné de la politesse compassée de Dharamsala, leurs tenues vestimentaires et leurs coupes de cheveux démodées, leur appétit carnivore et leur manières grossières, leur ignorance de l'hygiène et leur hébétude devant les objets technologiques de la modernité occidentale. On les a vus colériques, enclins à se battre, loin de l'image du bouddhiste non violent que les exilés de longue date affichent comme l'expression même de la tibétanité. Leur goût pour la musique et les films chinois, la présence de nombreux termes chinois dans leur vocabulaire (et pas seulement pour les objets modernes), leur refus du

15 A l'heure actuelle, selon le parcours, le tarif des passeurs varie entre soixante et trois cents euros.

16 Les garde-frontières tirent parfois à vue sur les fugitifs. Le dernier incident de ce type, qui s'est produit en septembre 2006 et a coûté la vie à une nonne, a été filmé en cachette par une équipe de télévision roumaine (vidéo accessible sur le site Youtube: http://www.youtube.com/watch? $v=i D d e W 2 q h Z 2 M$ ). Voir également Bruno Philip, « Des Tibétains cibles de soldats chinois dans l'Himalaya », Le Monde, 12 octobre 2006.

La période d'un an correspond à l'autorisation de séjour délivrée par le gouvernement indien (« Special entry certificate »), pour raison de pèlerinage ou d'éducation. Ils ne sont plus autorisés à séjourner au Népal, où ils sont confiés à un centre d'accueil du HCR, qui facilite ensuite leur acheminement vers l'Inde.

Le gouvernement en exil estimait en 2000 le nombre de Tibétains réfugiés (dans le monde entier) à cent trente mille, mais des observateurs occidentaux pensent que c'est bien en deçà de la réalité et qu'il faut plutôt en compter deux cent mille (Ginet 2000, p. 11). En Inde, ce sont bien sûr les « nouveaux venus » qui sont sans documents. 
manichéisme dans leur critique politique (qui tranche avec le discours simpliste de leurs prédécesseurs), et leurs propos mesurés sur la situation du Tibet, même s'ils y ont été des prisonniers politiques, persuadent les exilés d'Inde qu'ils ont subi un lavage de cerveau en Chine. Plus que jamais, pensent-ils, leur mission est de parler au nom de tout leur peuple sur la scène internationale. La mésentente s'est installée au fil des ans : au mépris des uns répond le mépris des autres (« ils parlent sans rien connaître ! »), à la méfiance des uns (« ce sont des espions ! ) répond le ressentiment des autres (« tant d'efforts, et aucune reconnaissance! »).

Les conditions de vie des nouveaux venus sont aussi devenues beaucoup plus précaires lorsque l'Inde a refusé d'accorder, dans les années quatre-vingt-dix, les fameux permis de séjour RC (registration certificate) aux réfugiés qui n'étaient pas nés sur son sol. Impossible, dès lors, de conduire une voiture, de faire du commerce, d'effectuer un travail rémunéré officiellement, de posséder un bien immobilier, ou de quitter le pays (Houston $2003: 224)^{19}$. Que faire alors ? Les « sans papiers » sont devenus très nombreux, et ils ne sont pas rares à avoir reçu de lourdes amendes et des peines d'emprisonnement, quand ce n'étaient pas des menaces d'expulsion vers la Chine.

Il faut toutefois souligner que cette seconde vague d'immigration, en dépit de ses difficultés, a néanmoins permis d'établir un dialogue entre les deux Tibet. Le Tibet de l'exil est devenu une ressource, tant matérielle que symbolique, pour les Tibétains de Chine, une alternative forte et structurée (même si elle a fait l'objet de nombreuses critiques) à la propagande de l'Etat chinois. C'est une échappatoire conceptuelle jouissant d'une audience internationale dont ne disposent pas les autres minorités ethniques.

C'est encore une autre vague d'immigration, cette fois vers l'Occident, qui va avoir les répercussions les plus importantes sur les conceptions que les Tibétains se font d'euxmêmes. L'exil vers l'Occident n'est certes pas récent, mais il ne commence comme mouvement de masse qu'en 1993. On notera que, dès le début des années soixante, une poignée de Tibétains ont été accueillis en Angleterre et en Amérique du Nord, pendant qu'environ mille deux cents d'entre eux ont été relocalisés dans les montagnes suisses ${ }^{20}$. L'ouverture de nombreux centres religieux a aussi entraîné une petite émigration. Mais celle-ci n'a pas suscité d'émulation pendant plusieurs décennies. Sans doute parce que l'Occident était perçu comme ambivalent: lieu d'abondance, certes, mais aussi de déchéance spirituelle et morale. Mais, en 1990, le Congrès américain vote la mise sur pied du Tibetan-U.S. Resettlement Project (TUSRP), c'est-à-dire l'octroi de mille visas d'immigrants à des Tibétains résidant en Inde et au Népal. Les candidats sont choisis par un système de « loterie $»^{21}$, organisée par le gouvernement-en-exil en 1992 (un quota de $10 \%$ a été réservé aux «nouveaux venus »). Les premiers immigrants arrivent aux EtatsUnis un an plus tard, non sans avoir suivi un stage préparatoire de quatre jours, destiné à leur rappeler leur devoir patriotique au moyen d'exercices intitulés, par exemple : « Je

19 Cette situation est fréquemment dénoncée par les nouveaux venus, mais il semblerait qu'ils puissent depuis peu obtenir à nouveau le RC : http://www.tibet.net/en/announcements/2006/circular.html (consulté le 27 mai 2007).

20 C'est un programme de la Croix-Rouge suisse qui a été effectif de 1960 à 1981 (Korom $1999: 5$ ).

21 Voir Hess (2006 : 86-88) pour les détails de la mise en place de ce projet, et les irrégularités qui ont été remarquées par certains réfugiés lors de la sélection par loterie. 
suis tibétain » (Yeh 2007). Avec le regroupement familial, les réfugiés sont rapidement plus de sept mille, rejoints également par plusieurs milliers de clandestins.

Parce qu'un nombre non négligeable des exilés sélectionnés proviennent de l'élite politique ou intellectuelle, et parce que tous renvoient de l'argent à leur famille en Inde, au Népal ou au Tibet (en omettant souvent de préciser leurs conditions de travail ${ }^{22}$ ), l'Amérique devient, aux yeux de la jeunesse tibétaine sous-employée ou désœuvrée en Asie, un «Shangri-la ${ }^{23}$ de substitution diamétralement opposé à la Chine » (Yeh 2007). L'aval politique stimule le processus : changeant radicalement de stratégie, le gouvernementen-exil encourage les migrants à opter pour la nationalité américaine (alors qu'il avait toujours empêché l'adoption de la nationalité indienne). Le but est d'acquérir un nouveau levier d'action (empowerment) sur la scène internationale, pour faire entendre « la voix de l'indépendance et de l'autonomie tibétaine », et faire des migrants de véritables « ambassadeurs culturels pour le futur du Tibet» (Hess $2006: 88$ ).

\section{Nouvelle forme de migration : l'asile politique}

Cette voie d'émigration saturée, c'est l'asile politique, qui s'impose rapidement comme solution alternative ${ }^{24}$. On se trouve alors quelques années après les manifestations indépendantistes de Lhasa (1987-1989), qui ont médiatisé en Occident l'image d'une Chine réprimant brutalement les droits de l'homme dans un Tibet récalcitrant. Les premiers dossiers sont convaincants et créent un précédent. Les Etats-Unis, l'Angleterre, l'Australie et le Canada, anglophones, sont des destinations privilégiées, mais les possibilités sont limitées. D'autres pays d'Europe de l'Ouest deviennent de nouvelles terres d'accueil : les Pays-Bas, la France, la Belgique, la Suisse et l'Allemagne, principalement ${ }^{25}$.

Il y a dix ans, il y avait en Belgique à peine une trentaine de Tibétains. Ils sont aujourd'hui environ mille deux cent cinquante. Il semblerait que tous, à l'exception de la petite dizaine d'entre eux qui sont arrivés en épousant un(e) Belge, soient inscrits dans une procédure d'asile, par contraste avec de nombreux autres migrants, notamment chinois, qui opèrent dans la clandestinité. Depuis 1990, environ mille deux cents demandes d'asile

22 Ainsi New York a-t-elle été rebaptisée en tibétain Mi-yog (esclavage) !

23 Lieu de fiction imaginé par James Hilton dans son roman Lost Horizon (1933). Shangri-la est le nom d'une vallée himalayenne paradisiaque, à l'écart du monde, peuplée de gens perpétuellement heureux. L'idée que le Tibet est réellement Shangri-la est le ressort principal de l'industrie touristique tant chinoise qu'occidentale.

24 Le phénomène semblerait avoir commencé en Europe, car Garratt (1997: 34) affirme que les premières demandes d'asile politique n'auraient eu lieu en Australie qu'en 1993 et aux Etats-Unis qu'en 1995, alors que le premier cas pour la Belgique date de 1990.

25 Le dernier recensement des Tibétains en exil remonte à 1998. Ci-dessous, les chiffres officiels du gouvernement-en-exil, auxquels il faudrait ajouter un grand nombre de clandestins : 7000 aux USA et au Canada, 1540 en Suisse, 640 en Europe du Nord-Ouest [Royaume-Uni principalement, puis France, Allemagne, et quelques rares Tibétains en Belgique], 110 en Scandinavie (principalement en Norvège) et 220 en Australie et en Nouvelle-Zélande. Relevons aussi la présence de mille Tibétains à Taiwan et de soixante au Japon (http://www.tibet.net/en/tibet/exile.html, consulté le 28 mai 2006). 
politique ont été enregistrées pour les Tibétains ${ }^{26}$. Les profils sont variés : la plupart ont moins de trente ans, les hommes (70\%) sont plus nombreux que les femmes $(30 \%)$, et environ $45 \%$ d'entre eux disent avoir été religieux à un moment de leur vie (proportion qu'il faudrait réduire à $20 \%$ du total si l'on ne prend en compte que les moines et nonnes ordonnés au Tibet et qui ont à peu près conservé leurs vœux après leurs exils successifs). La plupart des candidats réfugiés sont originaires de la région orientale du Kham. Ensuite viennent ceux de 1'Amdo au nord-est, et enfin une minorité du Tibet central ${ }^{27}$. Certains, déboutés, ont quitté le territoire, mais beaucoup d'autres sont arrivés par la voie du regroupement familial, ce qui explique qu'il y ait actuellement environ mille trois cents Tibétains en Belgique.

Pourquoi la Belgique ? Tous admettent qu'ils n'ont pas choisi ce pays, voire qu'ils n'en connaissaient même pas l'existence avant leur départ. C'est sans doute, comme pour tout mouvement migratoire de ce type, « un mélange de chance et de choix » (Havinga et Tötter 1999). Plus exactement, de chance pour les primo-arrivants, et de choix pour les suivants. Plusieurs facteurs expliquent la relative popularité du pays de destination : le prix du passeur ${ }^{28}$, ses connexions et son accès aux visas ${ }^{29}$, le taux d'acceptation des réfugiés dans le pays d'accueil, et enfin l'existence d'un secteur économique informel qui permette le travail au noir (id. : 53-54). La logique de la migration en chaîne fait que les suivants choisissent de s'installer en Belgique, au sein de réseaux familiaux ou amicaux, ce qui crée un effet boule de neige.

26 Les dossiers tibétains ne sont pas aisément quantifiables, étant donné qu'ils sont intégrés aux demandes enregistrées officiellement pour la Chine. A part un pic après les manifestations de la Place Tian'anmen (589 dossiers), les demandes chinoises ont stagné à une moyenne de vingt par an jusqu'en 2000. En se basant sur la consonance des noms, il est possible de déterminer que de 1990 (premier dossier tibétain en Belgique) à 2001, soixante-dix-neuf demandes de candidats réfugiés tibétains ont été enregistrées. A partir de 2002, les demandes ont fortement augmenté pour la Chine, avec une moyenne de deux cent cinquante demandes par an (201 en 2002, 284 en 2003, 212 en 2004, 304 en 2005, 155 en 2006). Environ 90\% de ces demandes sont le fait de Tibétains, les $10 \%$ restants couvrant des Ouighours, ainsi que des dissidents Han et des membres de l'organisation religieuse Falungong. C'est ainsi que j'estime le nombre total de dossiers tibétains à mille deux cents. Il semblerait que la France accueille davantage de réfugiés politiques Han, alors qu'il y en a comparativement très peu en Belgique. En revanche, ce pays n'accueille que peu de réfugiés tibétains (moins de trois cents jusqu'à aujourd'hui). Toutes ces informations proviennent du CGRA. Mentionnons enfin que la Belgique reçoit en moyenne, depuis 1988, quinze mille requêtes d'asile par an (si l'on excepte les deux pics d'environ quarante mille demandes en 1999 et 2000) et que, à titre de comparaison, l'Union européenne en reçoit chaque année plus de quatre cent mille (Granhag et al. $2005: 33)$.

27 Nombreux sont ceux qui se disent originaires du Tibet central, plus particulièrement des villages situés dans la partie occidentale de cette région, mais qui sont nés en exil. Ce sont en fait leurs parents qui sont originaires de cette région. La proximité de 1'Inde, donc la facilité relative avec laquelle ils ont pu fuir en 1959, explique qu'ils soient les plus nombreux parmi la population exilée.

28 Les réfugiés voyagent en avion, en utilisant de vrais ou faux passeports népalais ou indiens. En 2000, un passage se monnayait entre cinq mille et six mille euros. Aujourd'hui, le prix est monté à près de onze mille euros. Pour les Etats-Unis, il paraîtrait qu'il faut débourser vingt mille euros (informations des réfugiés euxmêmes).

29 Les filières seraient apparemment indiennes et népalaises (information des réfugiés), mais l'on ne peut exclure l'existence de filières tibétaines également. Toujours selon leurs dires, ils atterrissent généralement dans un pays voisin, avant d'être conduits en Belgique pour s'inscire à l'Office des Etrangers. 
Mais, si « arriver » apparaît aux Tibétains comme un aboutissement, leurs aventures ne font en réalité que commencer. La migration par le biais de l'asile politique a aussi ses contraintes, auxquelles les premiers arrivants n'étaient pas, c'est le moins qu'on puisse dire, préparés.

Leur volonté est bien sûr de s'acquitter au plus vite de leur dette (période qui s'étend de cinq à dix ans, parfois plus), donc d'obtenir le statut de réfugié politique. Ce statut est le sésame qui garantit le permis de séjour à durée indéterminée, le droit de travailler, le droit d'émarger à un Centre Public d'Aide Sociale (CPAS), si nécessaire, le très valorisé droit au regroupement familial, et enfin la possibilité d'obtenir la naturalisation après cinq ans.

Mais, confrontés pour la première fois à une procédure bureaucratique dont ils ne comprennent ni la raison d'être, ni les enjeux, ni le fonctionnement, les choses ne se passent pas avec la facilité prévue. Ils sont généralement refusés à la première audition à l'Office des Etrangers, et renvoyés devant le Commissariat Général aux Réfugiés et aux Apatrides (CGRA) pour un examen approfondi sur leur admissibilité au statut de réfugié politique ${ }^{30}$. Ils peuvent y être auditionnés deux fois. Le délai prévu entre les deux auditions est théoriquement de quelques mois, mais devant l'afflux surprise des Tibétains, il a fallu former des examinateurs, et les dossiers se sont accumulés, provoquant des délais d'attente de deux à trois ans (le retard est aujourd'hui résorbé en ce qui concerne les nouveaux arrivants). Pendant cette période, les candidats restent plongés dans une attente anxieuse, ne pouvant en principe ni travailler ni quitter le territoire (mais pouvant en revanche étudier les langues, ce que très peu d'entre eux s'attèlent à faire). Le taux d'acceptation des Tibétains par le CGRA n'est que de $18 \%{ }^{31}$. Ceux qui sont refusés peuvent présenter un dernier recours devant le Conseil du contentieux des Etrangers ${ }^{32}$, avec possibilité de pourvoi en cassation devant le Conseil d'Etat, mais le taux d'acceptation de ces demandes $\mathrm{y}$ est très faible (10\%). Cela implique qu'une large proportion des candidats, après la procédure d'asile, restent en Belgique «sans papiers » (depuis la réforme de 2006, ils peuvent toutefois introduire une demande de « protection subsidiaire $»^{33}$ ), quittent le pays pour tenter leur chance ailleurs (entreprise rendue plus difficile depuis le fichage par empreintes digitales), ou retournent, la tête basse, en Inde ou au Népal.

La procédure d'asile a été réformée en 2006. Cette première étape correspond à la procédure telle qu'elle existait au moment où ces matériaux ont été recueillis.

31 Information du CGRA. Ce taux est comparable au taux moyen d'acceptation en Belgique, toutes nationalités confondues. A titre de comparaison, le taux d'acceptation n'est que de $9 \%$ au Royaume-Uni (Good 2004 : 117). Changement en $2006: 30 \%$ des dossiers tibétains ont été approuvés, une augmentation qui a soudain placé les Tibétains parmi les nationalités au taux de reconnaissance le plus élevé au CGRA.

32 Avant la réforme de 2006, le recours se faisait auprès de la Commission Permanente de Recours des Réfugiés. Il y avait trois cents dossiers tibétains en cours dans cette juridiction en 2006.

33 Droit, renouvelable tous les ans pendant cinq ans, de séjourner sur le territoire de la Belgique et de bénéficier d'une aide médicale d'urgence, mais non assorti d'une aide financière (aide sociale, par exemple), ou d'une possibilité de regroupement familial. Après cinq ans, ils peuvent alors tenter une démarche de régularisation. 
La tâche des examinateurs du $\mathrm{CGRA}^{34}$ consiste à vérifier si le demandeur est bien un « réfugié » au sens où le définit la Convention de Genève (1951) ${ }^{35}$, auquel cas, en vertu du «principe de non-refoulement», le demandeur est autorisé à rester sous la protection de son nouveau pays de résidence. Cette définition, formulée au niveau du droit international, censée être uniforme pour les pays signataires, est pourtant floue, de même que la procédure exacte de certification des réfugiés. « Le droit d'asile est vide et le réfugié un concept juridiquement indéfini » (Valluy 2005 : 97). Ce sont donc les instances nationales qui sont les arbitres de la délivrance du statut de réfugié ${ }^{36}$.

Du fait de la position controversée du Tibet sur le plan du droit international (son incorporation à la République populaire de Chine en 1951 est toujours contestée par le gouvernement-en-exil tibétain, aidé de quelques avocats occidentaux), les cas tibétains posent problème au regard de la définition de l'ONU (Garratt 1997). De quelle nationalité sont les demandeurs ? Légalement, il est indiscutable que les Tibétains qui vivent ou qui sont nés au Tibet chinois après 1951 sont citoyens de la République populaire de Chine, mais qu'en est-il des exilés nés avant 1951 ? Il n'existait alors pas d'Etat tibétain reconnu internationalement, bien que les conditions soient réunies pour argumenter a posteriori de son existence de facto. Il semblerait que la jurisprudence internationale s'accorde à les reconnaître apatrides de facto, ainsi que leurs descendants, en dépit du fait que, de jure, la Chine proclame unilatéralement qu'ils sont des citoyens chinois par le seul fait de leur étiquette ethnique de « Tibétains » (id. : 30-33). Toutefois, le problème se complique par le fait que les demandeurs d'asile nés en exil masquent leur origine lorsqu'ils font leur demande. C'est au Tibet que s'exerce la répression, et donc ils prétendent être nés en Chine. Ce qui fait que dans leur dossier figure la mention « Pays d'origine : R.P. de Chine ", véritable claque, et même trahison, pour tout exilé scolarisé dans les valeurs patriotiques de Dharamsala. Si l'on ajoute que leurs noms et leur lieu de naissance sont systématiquement estropiés, au cours de la procédure, par une orthographe en pinyin très éloignée de la prononciation tibétaine ${ }^{37}$, les exilés démarrent leur requête d'asile par un très inconfortable sentiment de compromission politique.

34 Ce sont quatre ou cinq agents traitants flamands qui traitent les cas tibétains. En matière de réfugiés, il semblerait y avoir un partage informel du monde au sein des institutions belges : les Asiatiques, considérés comme majoritairement prédisposés à l'anglophonie, sont dirigés vers les structures flamandes, alors que les réfugiés en provenance d'Afrique francophone, d'Amérique Latine, ou parlant une langue latine sont orientés vers les francophones.

35 «...) le terme 'réfugié' s'appliquera à toute personne qui, (...) craignant avec raison d'être persécutée du fait de sa race, de sa religion, de sa nationalité, de son appartenance à un certain groupe social ou de ses opinions politiques, se trouve hors du pays dont elle a la nationalité et qui ne peut ou, du fait de cette crainte, ne veut se réclamer de la protection de ce pays ; ou qui, si elle n'a pas de nationalité et se trouve hors du pays dans lequel elle avait sa résidence habituelle à la suite de tels événements, ne peut ou, en raison de ladite crainte, ne veut y retourner » (Convention [1951] et protocole [1967] relatifs au statut des réfugiés, p. 16, consultable sur le site du HCR : http://www.unhcr.fr/cgi-bin/texis/vtx/basics/opendoc.pdf? tbl=BASIC $S \& i d=41 a 30 b 9 d 4$, consulté le 3 février 2007).

36 Les traités de Maastricht (1993) et d'Amsterdam (1999) constituent toutefois une tentative de mise au point d'une politique européenne commune en matière de réfugiés.

37 Par exemple, Tashi devient en pinyin Zhaxi, Phuntsog - Pingcuo, Öser - Wosei, Dölkar - Zhuoga, Thinley Jinlai, etc. Il en va de même pour les toponymes : Chamdo - Changduo, Gyantse - Jiangzi, Labrang Labuleng, etc. 
Ceux dont le statut n'est pas reconnu sont en principe expulsables vers leur pays d'origine. Expulser des Tibétains nés en Inde vers la Chine serait un comble, et bien sûr, cela ne se produit jamais. Lorsqu'ils reconnaissent venir d'Inde, ils ne risquent pas l'expulsion, puisque la Belgique n'a pas d'accord bilatéral d'extradition avec l'Inde (laquelle s'accommode d'ailleurs très bien du départ de ces réfugiés). Et lorsqu'ils viennent de Chine, étant donné le risque avéré de représailles qu'ils encourent en tant que Tibétains, et en vertu du principe de non refoulement, ils ne risquent pas non plus l'expulsion. L'une des particularités des réfugiés tibétains en Belgique est donc que, d'où qu'ils soient originaires, ils sont en pratique inexpulsables. Commencent alors pour la grande majorité d'entre eux les vicissitudes de la vie de "sans papiers », avec l'espoir d'une régularisation à long terme... une catégorisation sociale précaire qu'ils n'avaient pas anticipée en payant leur passeur quelques années auparavant.

Le contexte historique et procédural de l'asile politique étant esquissé, examinons à présent les lignes de partage révélées par les récits de ces demandeurs d'asile.

\section{Le témoignage de procédure d'asile}

- Qu'avez-vous fait et pourquoi êtes-vous partie de votre couvent?

- Moi, en fait, je n'ai rien fait. J'étais juste dans la même cellule que Paldron. Le frère de Paldron venait de revenir d'Inde. Il avait ramené quelques livres et discours du Dalai Lama. Il avait aussi des cassettes, mais c'était trop risqué pour nous. Nous nous passions les livres en cachette. Nous les lisions sous les couvertures, le soir, à la lampe de poche. - A quoi ressemblaient ces livres?

- Cétaient des petits livres avec une couverture verte. Je les apprenais par cour. Un nectar pour le cœur. Des paroles de sagesse. Paldron n'avait pas bonne réputation au couvent. Elle avait été expulsée plusieurs fois parce qu'elle refusait de renier le Dalai Lama, mais la supérieure avait su s'arranger à chaque fois avec la police. Paldron, c'était une vraie nationaliste. Elle détestait les Chinois. Tous les Chinois. Elle avait perdu son père et un oncle dans les manifestations de Lhasa. Ils étaient morts en prison. Ce n'est pas comme les autres, celles qui se taisent pour ne pas avoir d'ennuis. Elle, elle aimait ça, courir au devant des ennuis. En fait, je trouvais que ça ne servait à rien de faire ça. Quand l'équipe de rééducation patriotique est revenue nous interroger au couvent, on nous a annoncé qu'il fallait expulser cinq nonnes parce que nous étions trop nombreuses. Ils ont nommé Paldron en premier. Elle a crié que c'était injuste. On s'est levées et on a fait barrage. Alors on a commencé à se battre avec eux.

- Combien y avait-il d'hommes dans cette équipe?

- Oh, euh, trois. Non, quatre. Oui, ils étaient quatre avec le chauffeur. On a crié et le ton est monté. Il y en a plusieurs qui criaient "Longue Vie au Dalai Lama!", "Que le vrai Panchen Lama soit reconnu! », " Le Tibet est indépendant! ». J'avais très peur. Alors j'ai commencé à crier aussi. Ils sont sortis et ils se sont rabattus dans leur voiture. Ils sont partis en trombe. Ils sont revenus une demi heure plus tard avec la police. On savait qu'ils allaient fouiller nos cellules. On avait caché les livres, mais on savait qu'ils allaient les trouver. Alors, quand on a vu les voitures de police, on s'est enfuies. On était quatre. Paldron, elle, ne pouvait pas partir, parce qu'elle était handicapée et elle boitait d'une jambe. Nous avons couru, nous avons passé le col. Puis, on s'est séparées. Je ne me suis pas retournée. Je ne suis pas allée dans ma famille. Je me suis cachée dans la famille de mon oncle pour la nuit. 
C'est lui qui m'a dit que je devais fuir [en Inde], et il m'a donné des pierres [semiprécieuses] pour payer le passeur. Il m'a dit aussi que Paldron avait été arrêtée avec d'autres nonnes.

Autre témoignage :

- C'était le 10 décembre. Nous étions quatre: Lobsang mon jeune frère, Tashi et Samten, nos voisins. Nous avons préparé nos affiches la veille.

- Vous savez donc écrire?

- Non, moi, je ne sais pas écrire, parce que je ne suis allé que jusqu'en troisième classe. Nous avions demandé au frère de Tashi, qui écrit bien. C'est lui qui a écrit les slogans sur les affiches.

- Quels étaient les slogans qu'il avait écrit? Pouviez-vous les lire?

- Oui, je sais lire! Mais je ne sais pas écrire. Il avait écrit : "Longue Vie au Dalai Lama!", "Que les droits de l'homme soient reconnus au Tibet!", "Libérez le plus jeune prisonnier politique du monde! " [le Panchen Lama], "La Chine rouge hors du Tibet! », "Sauvez l'environnement du Tibet!».

- Combien y avait-il d'affiches?

- J'en avais dix, mon frère en avait huit, Tashi et Samten en avaient six chacun. Chacun avait des affiches avec des slogans différents.

- Et pourquoi aviez-vous prévu de coller vos affiches ce jour-là?

- C'est le jour anniversaire de la remise du prix Nobel de la paix au Dalai Lama. Il fallait qu'on manifeste notre mécontentement. Le gouvernement chinois est vraiment mauvais avec le peuple tibétain. Nous savions qu'il allait y avoir une visite $d u$ chef de district au village ce jour-là. Alors on a voulu montrer qu'on n'était pas d'accord avec la politique du gouvernement et on a voulu coller des affiches sur les murs du bureau du parti, sur les grilles de l'école, sur le mur du bureau des affaires agricoles et sur la façade d'un commerce, juste devant le bureau du parti. Nous nous étions réparti entre nous les lieux d'affichage.

- Qui est le chef du gouvernement chinois?

- Euh... Je ne sais pas.

- Quel type de papier et quelle encre ont été utilisés pour réaliser ces affiches?

- On avait acheté le papier au commerçant et ...

- De quelle dimension était le papier?

- Euh, comme ça (il montre avec les mains). Et l'encre, on l'avait volée à l'école.

- Le papier était de quelle couleur?

- Blanc.

- Et la colle?

- On n'avait pas de colle. On a utilisé de la tsampa [farine d'orge grillé, mets de base des Tibétains] mouillée.

- Est-ce qu'on vendait de la colle dans votre village?

- Je ne sais pas. Je n'en ai jamais vu.

- Et ensuite, que s'est-il passé?

- Ensuite, ça ne s'est pas passé comme on avait prévu. On a commencé à coller nos affiches très tôt, le soleil n'était pas encore levé, et ...

- Quelle heure était-il?

- Oh, je ne sais pas, je ne sais pas lire l'heure. C'était encore la nuit.

- ... et alors, quelqu'un nous a vus. Je ne sais pas exactement qui nous a vus, peutêtre le gardien du bureau du parti. C'est moi qui collais les affiches là. Tout à coup, 
on a entendu les sirènes de la police. J'ai crié à Tashi et à Samten de fuir, parce qu'ils étaient encore petits [quatorze et seize ans], et ils couraient moins vite. Moi, j'ai pris mon frère par le bras et on a couru.

- De quelle couleur sont les uniformes de la police?

- Kaki.

- Comme ceux de l'armée?

- Euh... oui.

- Où êtes-vous allés?

- On a couru jusqu'au bout du village, puis on a grimpé le col. Les policiers nous ont poursuivis en voiture, mais pas quand on a monté à flanc de montagne. On a couru, couru, sans se retourner. On a marché toute la journée. On dormait en montagne, entre deux rocs, puis on marchait le jour suivant. On est allés se cacher chez notre oncle. J'avais très peur, parce que notre famille avait certainement des problèmes à cause de nous. Ils étaient pour sûr en train de se faire interroger. J'étais si triste pour ma mère. On ne les avait pas prévenus. Je ne l'avais pas revue. (...) [Il pleure]. Mais maintenant, il fallait fuir. Pour le bien de tout le monde, il fallait fuir. Alors mon oncle nous a confiés à un commerçant de peaux et de laines qui passe fréquemment le col vers le Népal, juste de l'autre côté. Nous étions déguisés en gardiens de troupeaux et nous avons passé le col avec lui. Je ne sais pas ce qui est arrivé à Tashi et à Samten. Je ne sais pas ce qui est arrivé à ma famille. Il n'y a pas le téléphone au village.

Les extraits qui précèdent constituent le cœur du dossier d'un candidat : les activités qui prouvent que ses craintes de persécution sont fondées. Pourtant, ce moment clé n'intervient généralement qu'en milieu ou en fin d'audition. Une audition au CGRA dure entre deux heures trente et quatre heures, largement dévolues à certifier l'identité du candidat: la composition de sa famille, son éducation, son parcours, son lieu de résidence, son mode de vie, et enfin ses activités politiques. Comme les preuves tangibles (documents officiels ${ }^{38}$, preuve des mauvais traitements infligés, possibilité d'appeler les persécuteurs comme témoins) sont impossibles à réunir, et comme le temps imparti pour chaque interrogatoire est court, c'est sur le témoignage du candidat lui-même que repose tout son dossier. A défaut d'avoir les capacités de se prononcer sur la vérité de ce témoignage, les agents traitants en évaluent la crédibilité (Granhag et al. 2005 : 46). Il faut souligner que les exigences juridiques en matière de preuve sont abaissées (« low standard of proof », Good 2004 : 114) dans le cas de la procédure d'asile, en raison de la difficulté d'apporter des preuves concrètes et de l'importance des conséquences de la décision du Commissariat.

Le Commissariat décrit lui-même ses critères d'évaluation: «Après l'audition, l'agent traitant examine la crédibilité du récit et si les déclarations correspondent aux critères de la Convention de Genève. Il compare les déclarations successives et place

C'était surtout le cas pour les premières centaines de dossiers tibétains, à l'époque où cette recherche a été menée. A l'heure actuelle, l'évaluation des dossiers fait une part plus large aux documents officiels, qu'ils soient carte d'identité chinoise (certaines fausses cartes d'identité chinoises fabriquées en Inde ont néanmoins été débusquées), documents du centre de réception des réfugiés de Katmandou, photographies personnelles, ou documents de l'association Gu-chu-sum de Dharamsala, qui prend en charge les exprisonniers politiques. 
le récit subjectif dans un contexte objectif $\aleph^{39}$. Les deux points principaux qui président à l'accréditation du témoignage sont donc deux critères de cohérence : une cohérence interne aux déclarations du demandeur, et une cohérence entre ces déclarations et le contexte qu'elles décrivent (plausibilité par rapport à un ensemble de savoirs vérifiables, tels des variables géographiques, politiques, sociologiques ou historiques sur le Tibet). Les agents traitants ne s'attachent nullement, pour détecter des mensonges éventuels, à observer le comportement non verbal (voix plus faible ou plus aiguë, longues pauses, mouvements des bras ou des jambes, évitement du regard, sourire, nervosité, ou autres croyances stéréotypées sur la tromperie), car ils partent du principe que les stratégies d'artifice sont culturellement spécifiques, donc difficilement interprétables (Granhag et al. $2005: 30$ ). De plus, la médiation de l'interprète interfère dans le face-à-face et introduit des pauses dans l'échange. C'est donc sur la seule base du contenu de leurs récits que les candidats vont être jugés, plus exactement, sur leur " rationalité narrative " (Hesford 2004). Or, pour objective et a-culturelle que cette rationalité puisse paraître, elle n'en repose pas moins sur un ensemble de conventions et de savoir-faire, sur une « compétence narrative » qui échappe le plus souvent aux demandeurs d'asile provenant de cultures éloignées des nôtres (Dobson 2004 : 131-134).

L'ajustement culturel qui a dû se mettre en place tant du côté des demandeurs tibétains que des interrogateurs belges a été long et difficile. Il a fallu plusieurs dizaines, peut-être plusieurs centaines de dossiers pour aboutir à un processus d'interrogatoire mutuellement satisfaisant. Pourquoi est-ce si difficile de faire parler les Tibétains, alors que, si l'on accepte une comparaison un peu schématique, on peine à faire taire les Congolais ? Pourquoi une telle réticence à s'exprimer? De toute évidence, pas uniquement pour cacher un mensonge, car des prisonniers politiques «authentiques » (environ une quinzaine en Belgique), c'est-à-dire répertoriés comme tels dans des listes établies par le Tibetan Centre for Human Rights and Democracy et l'organisation Gu-chu-sum de Dharamsala, faisaient partie de ces candidats étrangement peu diserts. Il faut souligner que, entre eux, les Tibétains sont bien sûr d'habiles et abondants parleurs, et que la rationalité argumentative ne leur fait nullement défaut (que l'on songe aux disputes dialectiques entre moines ou aux combats de chants et généalogies entre villageois). Ils ont grandi dans une civilisation où la belle parole joue un rôle social fondamental, où le pouvoir de convaincre n'est pas celui de l'enchaînement logique, mais plutôt celui d'apaiser, de rassembler par des références aux codes, dictons et valeurs du passé. Inversement, les Tibétains, mal informés sur la procédure et peu curieux de son fonctionnement, n'ont pas compris la raison des questions du CGRA. Ils se sont révoltés par ce qu'ils ont perçu comme un traitement injuste de leurs dossiers, "à la tête du client", en fonction des connaissances préalables ou de la persévérance de l'interrogateur du jour. Il faut aussi ajouter d'importants problèmes de traduction dans les premières années : le tibétain est une langue fractionnée en plusieurs dialectes, ce qui a rendu la compréhension avec l'interprète parfois très difficile. Plusieurs candidats ont ainsi été atterrés de découvrir que leurs propos avaient été tronqués lors du premier interrogatoire (l'aune à laquelle sont rapportées toutes leurs déclarations ultérieures). Comment peuvent-ils dès lors corriger ce récit, puisque l'exercice de cohérence auquel ils sont astreints est de le répéter

39 «Le traitement des dossiers, » site du CGRA (http://www.belgium.be/cgra, consulté le 10 mai 2007). Italiques ajoutées. 
à l'identique ? L'évaluation de la rationalité narrative des candidats a en réalité rencontré plusieurs types de problèmes, qui vont être présentés successivement.

D'abord, le témoignage d'asile est une forme de présentation de soi fondée sur la victimisation. Ce récit autobiographique visant à prouver une persécution de nature collective (une violation des droits de l'homme) est très éloignée des conceptions de l'identité personnelle et des conventions discursives du Tibet traditionnel ${ }^{40}$. Ceux qui ont effectivement été victimes de mauvais traitements ont immédiatement rencontré un frein culturel à l'exposition de leurs souffrances. Non pas tant par pudeur qu'en raison de conceptions relatives à la manière " interdépendante » dont s'enchaînent les phénomènes (tendrel, concept d'origine bouddhiste qui a trouvé un large écho dans la pratique sociale). Il ne porte pas chance de raviver la mémoire des traumatismes passés, car cela leur redonne un pouvoir d'action sur le présent. C'est pourquoi les observateurs occidentaux qui voulaient venir en aide aux premiers réfugiés en 1959 se sont heurtés à un mutisme relatif, de même les médecins occidentaux qui ont examiné d'anciens détenus politiques passés en Inde dans les années quatre-vingt-dix (Crescenzi et al. 2002). Au risque d'un parallèle culturel audacieux, on pourrait dire que, comme les personnages de Shakespeare, les Tibétains sont enclins à penser que « gémir sur un malheur passé, c'est le plus sûr moyen d'en attirer un autre ${ }^{41} »$. Il ne faudrait pas en inférer, néanmoins, qu'aucune expérience négative n'est jamais partagée, ou qu'aucune mémoire des traumatismes n'est transmise entre les générations. Mais elles n'empruntent pas cette "rhétorique du témoignage » (Hesford 2004) pratiquée dans nos sociétés, distincte du langage ordinaire que l'on produit dans des circonstances informelles. Toutefois, depuis une vingtaine d'années, les Tibétains de l'exil ont trouvé les moyens de s'adapter à cette rhétorique, en la voyant comme le seul moyen de se faire entendre sur la scène internationale. Pour l'efficacité de l'activisme politique, c'est le registre affectif qu'il a fallu mobiliser (McLagan 2002). Il a fallu s'ouvrir, s'exposer, trouver des victimes qui voulaient parler, apprendre les codes tacites qui régissent l'efficacité médiatique, scénariser les événements plutôt que les décrire (emplotment, Dobson 2004) pour concentrer le propos, fournir des images spectaculaires et marquer les esprits. C'est l'empathie suscitée par les témoignages qui allait devenir le levier de l'activisme, et non pas, comme ils l'avaient cru jusqu'alors, la simple conviction que leur cause était bien fondée. Certains Tibétains se sont pliés avec plus de facilité que d'autres à cet exercice de communication : Päldèn Gyatso (1997) a fait peut-être le tour du monde avec le récit de ses trente-deux années de détention. Mais il est de nombreux autres témoignages tibétains qui n'ont pas été retenus en raison de leur non conformité au style canonique du « réalisme traumatique » (Hesford 2004 : 105) en Occident (McLagan 2002). Ainsi, les demandeurs d'asile nés en exil ont-ils absorbé ce mode discursif façonné pour les Occidentaux, au contraire de leurs compatriotes nés au

40 Il faut toutefois mentionner qu'à l'ère moderne, les « récits d'amertume » (chin. suku), bien connus de ceux qui vécurent la période de maoïsme radical (jusqu'à la mort de Mao en 1976), sont également des récits de victimisation collective fondés sur la « preuve » autobiographique : les individus des classes « libérées » par le communisme étaient tenus, pour témoigner leur loyauté au nouveau régime, de dénoncer publiquement et de manière répétée les abus de leurs anciens exploiteurs. Ce type de discours a néanmoins disparu avec la fin de la pratique révolutionnaire, et l'on peut dire qu'il est aujourd'hui inconnu des jeunes Tibétains tranche d'âge largement majoritaire parmi les demandeurs d'asile. 
Tibet. Lors de l'interrogatoire au Commissariat, ils ressortent, de manière irréfléchie, ce mode de discours, et même s'émeuvent, souvent de manière tout à fait sincère, avec des larmes parfois, des avanies subies par leur peuple. A leurs yeux, leur expérience politique est « vraie », leur investissement est total (au contraire, pensent-ils, des Tibétains du Tibet qui, opportunistes, ne seraient plus politisés), à défaut de pouvoir prouver la vérité de leur histoire personnelle. Or, la procédure d'asile se fonde sur une notion de vérité qui ne fait aucun cas de la compréhension personnelle des candidats de ce qui fait la vérité leur expérience (Good 2004 : 113). Le but de l'interrogatoire n'est pas de susciter l'empathie, mais de persuader grâce à une construction logique sans faille et à l'absence de mensonge avéré. Ici aussi, l'expérience doit être reconstruite par le biais d'une narrativisation, mais les conventions en sont différentes. C'est ainsi que de nombreux candidats ont mis du temps à comprendre pourquoi l'interrogateur essayait si ardemment de débusquer leurs contradictions et infidélités narratives. Pourquoi fallait-il donc répéter plusieurs fois de quelle couleur étaient les meubles de la cellule, ou en quelle matière était la porte ? Quel type d'encre et de colle ont été utilisés ? Est-ce que la fenêtre s'ouvrait vers l'intérieur ou vers l'extérieur? Quel est le jour exact du départ de Lhasa, mardi ou mercredi ? Combien coûte le passeur de la rivière : trois ou quatre yuans? Nombreux sont ceux qui ont été mis en difficulté par ces détails. Lors du premier interrogatoire, ils ont parfois répondu au hasard, juste pour avoir quelque chose à dire, même s'ils avaient vécu ces événements, mais sans prendre garde de mémoriser leur réponse.

La crédibilité générale d'un témoignage dépend non seulement de cette cohérence, mais aussi de l'abondance et de la précision des détails. Mieux on est capable de décrire, plus on donne l'impression de se baser sur du vécu. On serait tenté de croire que les Tibétains de l'exil sont, en situation d'interrogatoire, moins loquaces que ceux du Tibet, vu leur ignorance des conditions de vie au Tibet lui-même. Pour nombre d'entre eux, c'est le cas, et leur ignorance devient criante. Ce qui surprend le plus l'observateur extérieur n'est pas leur déficit en connaissances de base (histoire, mode de vie, géographie la plus élémentaire du Tibet), qui contraste avec leur activisme politique vigoureux : on pourrait en rejeter la faute sur le curriculum scolaire de l'exil. C'est plutôt leur absence totale de curiosité, surtout après leur arrivée en Belgique, où ils pourraient se documenter ou poser des questions aux Tibétains du Tibet, alors qu'ils doivent consolider leur dossier au Commissariat. Ceux-là se disent souvent fils de paysans, n'étant jamais sortis de leur village (ce qui justifie leurs lacunes en géographie régionale), à peine éduqués par un voisin ou par quelques années dans une école locale (raison de leur ignorance du chinois ${ }^{42}$ ). Ils se retranchent habilement, à chaque question difficile, derrière un « je ne sais pas » qui sousentend « on ne m'a pas donné la possibilité de savoir», pour incriminer l'Etat chinois (qui marginalise effectivement les Tibétains des campagnes) plutôt qu'eux-mêmes. Ceux-là ont bien sûr reçu en Inde une éducation avancée. Au CGRA, ils doivent rester vigilants et maintenir leur rôle de composition jusqu'au bout : l'analphabétisme (alors qu'ils déclarent une activité politique d'affichage ou de distribution de tracts), le manque de culture générale (alors qu'ils parlent de concepts abstraits tels les droits de l'homme

42 Cette ignorance est néanmoins plausible, même après cinquante années de présence chinoise. J'ai rencontré dans des villages, qui ne sont pas si éloignés de Lhasa, de jeunes Tibétains qui ne connaissaient pas le chinois. 
ou la protection de l'environnement), et bien sûr l'ignorance absolue de l'anglais (ils se trahissent parfois en écoutant directement l'interrogateur et non l'interprète, ou alors en laissant échapper un soupir de désaccord sur une traduction). Mais il ne faudrait pas généraliser et penser que, a contrario, la volubilité serait un trait distinctif des Tibétains du Tibet. Eux aussi ont été taciturnes, surtout dans les premiers temps. Deux raisons semblent l'expliquer : moins familiers que les autres avec les institutions occidentales, ils ne savaient pas dans quelle mesure faire confiance aux interrogateurs, et à l'Etat belge en général : leurs propos allaient-ils être répétés à l'ambassade de Chine, entraînant menaces et répercussions sur les membres de leur famille restés au pays ? Assurément, il fallait en dire le moins possible, et surtout ne nommer ni les gens ni les lieux (un choix qui était évidemment à l'opposé d'une stratégie de crédibilité). Enfin, certains de ces candidats ont éprouvé au quotidien un tel fossé d'incompréhension entre Belges et Tibétains qu'ils se sont persuadés que les interrogateurs ne les comprendraient pas. Par où commencer pour expliquer leur mode de vie et les raisons de leurs actions? Mieux valait rester économe en paroles, ce qui nourrissait aussi les doutes du CGRA.

Un autre problème, et non des moindres, rencontré par les agents traitants concerne l'identification précise des individus interrogés. Les trois paramètres fondamentaux de l'identité que sont en Occident le nom, le lieu et la date de naissance faut défaut chez les Tibétains. Non pas pour des raisons administratives (les actes de naissance ou de mariage n'existent effectivement pas), mais pour des raisons culturelles. D'abord, pas de patronyme au Tibet, sauf dans certaines régions et dans les familles aristocratiques. Les Tibétains portent deux noms (parfois un seul) attribués le plus souvent par un religieux pendant la petite enfance, et qui sont extraits d'un répertoire assez limité. De manière schématique, on pourrait dire qu'ils portent tous le même nom. Il est en outre fréquent qu'une personne soit connue sous plusieurs noms, soit qu'elle ait changé de nom au cours de sa vie (à l'enfance, ou suite à une maladie, à la prise de vœux monastiques, ou pour masquer un passé criminel), soit qu'elle cumule plusieurs noms (chinois et tibétain, ou nom et surnom). Les documents que certains demandeurs versent à leur dossier ne sont pas toujours au nom qu'ils ont renseigné. Ce n'est pas parce qu'ils chercheraient à masquer leur refoulement d'autres pays européens, comme le suspecte le Commissariat. Ensuite, le jour exact de la naissance des enfants n'a pas d'importance culturelle. Parfois, c'est l'année elle-même qui est inconnue, ou approximative. De nombreux Tibétains ne peuvent dire leur âge ni celui de leurs enfants, surtout à la campagne. Et encore, il arrive qu'ils renseignent délibérément une année de naissance erronée pour des raisons superstitieuses (pour éviter la magie noire, principalement). De plus, les repères temporels sont fixés dans le calendrier lunaire tibétain pour la plupart des paysans, éleveurs et religieux, une équivalence dans le calendrier occidental malaisée à calculer. Enfin, concernant le lieu de naissance, on comprend aisément les modifications des candidats nés en exil, mais même ceux qui sont nés au Tibet changent leur lieu d'origine: souvent parce que la rumeur veut que « ceux de Lhasa », ou « ceux de l'Amdo » aient plus de chance d'être reconnus réfugiés. Lors du premier interrogatoire, il faut bien déclarer une identité compréhensible par l'employé qui consigne leur déposition, c'est pourquoi ceux qui ne connaissent pas leur date de naissance en inventent une, qu'ils oublient parfois aussitôt. Ou alors, à des fins mnémotechniques, ils placent tous les événements de leur récit à la même date, en changeant seulement les années : un $1^{\text {er }}$ janvier, lors de leur prétendu anniversaire, ou lors des dates-clés du calendrier tibétain. Les repères temporels farfelus de ces récits ont 
longtemps éveillé la suspicion des agents traitants, et pourtant, certains étaient le fait d'anciens détenus politiques reconnus.

Enfin, la dernière pierre d'achoppement des agents traitants concerne leur difficulté à « placer le récit subjectif [des candidats] dans un contexte objectif », pour reprendre les termes du Commissariat. Comment juger la plausibilité de certaines déclarations qui semblent contraires au sens commun (l'ignorance du chinois alors que l'on a grandi en Chine, la méconnaissance des hommes politiques, l'ignorance du prix de certaines marchandises, une répression politique décrite comme systématique et omniprésente, par exemple) ? Quelle est le contexte de vie quotidien des Tibétains ? Quelle est la nature des pressions qui sont exercées sur eux ? Qu'est-on en droit d'attendre de leur part comme connaissances minimales? Vu la notoire rétention d'informations qui caractérise la Chine contemporaine (et une opacité totale lorsqu'il s'agit de questions « sensibles », lot quotidien des anthropologues, juristes ou autres employés d'ONG), on est bien en peine de trouver des comptes rendus officiels d'échauffourées entre police et population, ou des listes de prisonniers politiques. Ensuite, malgré la popularité relative du Tibet dans le grand public et les images qui circulent dans les médias, on connaît en fait peu de choses sur la vie quotidienne. Enfin, au fil des auditions de Tibétains provenant de tous horizons, les examinateurs se sont rendu compte de l'énorme diversité des vécus quotidiens, voire de leur incommensurabilité : entre les trois régions tibétaines traditionnelles, entre les grandes villes et les petites bourgades, et entre celles-ci et les villages, entre ceux-ci et les campements nomades, entre ceux qui ont été scolarisés en chinois et ceux qui ne l'ont pas été, entre ceux qui se sont assis ne fût-ce que quelques années sur les bancs d'une école et ceux qui ont suivi un cursus monastique en marge de la société, et puis entre tous ces exemples-types et les cas hybrides, il n'y a aucune mesure. Il n'y a pas « un » Tibet, mais une myriade de Tibet(s), et les dossiers ont dû être évalués au cas par cas. Les primoarrivants ont eu sur les suivants un grand avantage, celui d'une carte blanche quasi totale, puisque rien ne pouvait recouper leurs déclarations. Chemin faisant, à travers les centaines de dossiers traités, les examinateurs ont aussi construit leur expertise, en repérant les « bons » dossiers, en posant à ces candidats des questions de contexte général (sur la vie quotidienne des paysans, nomades, moines ou citadins selon les cas, sur l'expérience de l'arrestation, du jugement ou de la prison). Les demandeurs sont ainsi devenus, malgré eux, des informateurs. Et les agents traitants ont accumulé sur certains sujets plus de connaissances que les Tibétains nés en exil...

\section{Un imaginaire politique différencié}

Lorsqu'ils abordent les (vraies ou fausses) raisons politiques qui ont motivé leur fuite, les candidats, qu'ils proviennent de l'exil ou du Tibet, fondent leur récit sur des éléments récurrents, qui sont avérés dans les manifestations au Tibet depuis une vingtaine d'années. D'abord, le point de départ de leur protestation est un moment ou un endroit (comme un monastère ou un lieu de pèlerinage) culturellement marqués comme typiquement tibétains. C'est souvent le nouvel an, le jour de l'anniversaire du Dalai Lama (le 6 juillet), ou alors une date privilégiée du calendrier rituel ${ }^{43}$, quand ce n'est pas le

43 Le quinzième jour du quatrième mois (commémoration de la naissance, de l'éveil et de la mort du Bouddha), le quatrième jour du sixième mois (jour des dieux protecteurs du Tibet), le vingt-cinquième jour du dixième mois (jour de la mort du grand réformateur religieux Tsongkapa), par exemple. 
jour anniversaire de la fondation d'un édifice religieux local. Mais le choix des référents politiques contemporains tibétains ${ }^{44}$ semble être un trait distinctif, et donc disqualifiant, des demandeurs nés en exil. Ces événements ne sont bien sûr pas commémorés au Tibet. Ils n'y sont pas devenus des dates signifiantes, par opposition aux grandes manifestations annuelles qu'ils suscitent à Dharamsala.

De la même manière, les activités politiques qu'ils prétendent avoir menées se déclinent invariablement autour des mêmes thèmes (relevons d'emblée que les faux témoignages ne sont pas l'apanage des exilés, puisque la plupart des Tibétains du Tibet n'ont pas fui, eux non plus, suite à une confrontation politique avec menace d'emprisonnement). Chaque récit est centré, immuablement, sur la personne du Dalai Lama, «le principe espérance » (Assayag 1989: 71) qui soutient symboliquement tout son peuple par-delà ses fractures. Les protestations sont généralement locales et de petite ampleur : acheminer et transmettre des écrits, des photos ou des cassettes du Dalai Lama, écouter l'une des radios émettant depuis l'Occident, confectionner ou hisser un drapeau tibétain, et enfin, activité la plus fréquemment invoquée, réaliser et coller des affiches portant des slogans politiques ${ }^{45}$. Mais c'est dans le choix des slogans que réapparaît le fossé qui sépare les deux groupes de Tibétains. Certaines revendications portent immanquablement le sceau de l'exil, comme les références aux droits de l'homme, aux prisonniers politiques qu'il faut libérer, à la préservation de la nature, ou au Tibet comme zone de paix mondiale. Les revendications citées par les Tibétains du Tibet sont plus concrètes et plus spécifiques (le refus de reconnaître tel enfant choisi par le parti comme réincarnation d'un maître religieux, par exemple). Tous, néanmoins, mentionnent des slogans sur l'indépendance du Tibet et l'expulsion des Chinois, à l'énoncé desquels ils ont fréquemment une forte réaction émotive, spontanée, qui semble les surprendre eux-mêmes. Autre élément récurrent : la rancœur indiscriminée contre « les Chinois », souvent suite à la mort ou à l'emprisonnement de membres de la famille.

Il est difficile, à partir de ces récits comportant une majorité de faux témoignages, de se faire une idée de la nature exacte de la répression politique qui pèse sur les Tibétains. La situation n'est clairement pas celle d'un conflit ouvert, ni d'un encadrement total, systématique, militaire, comme cela a pu être le cas dans le passé. On ne peut davantage affirmer qu'une majorité de Tibétains sont aujourd'hui impliqués politiquement, ou engagés dans ce que nous nommerions la résistance ${ }^{46}$. On se trouve ici dans une situation chronique de marginalisation et chacun essaie vaille que vaille de s'en sortir comme il

44 Le 10 décembre (commémoration du Prix Nobel du Dalai Lama) ou le 10 mars (commémoration du soulèvement de Lhasa), pour les plus importants. Relevons néanmoins que certains récits, le plus souvent des Tibétains du Tibet, prennent pour point de départ des commémorations politiques chinoises (le $1^{\mathrm{er}}$ octobre, ou le 23 mai, anniversaire de la signature de l'accord sino-tibétain de 1951), occasions de réactions de désapprobation. Inversement, ces dates ne sont pas commémorées en exil.

45 Le collage d'affiches n'est assurément pas, de par sa fréquence effective, le « sport national tibétain », comme l'ont formulé ironiquement certains agents du CGRA, mais il n'est pas rare de lire l'arrestation de ce type de manifestants, comme à Ganze, dans l'est du Tibet, en juin et août 2006 (http://www.rfa.org/ english/news/2006/08/18/tibet_arrest/, consulté le 25 mai 2007). réducteur pour qualifier les diverses attitudes tibétaines face au pouvoir en place. 
peut. Cette marginalisation prend plusieurs formes et touche les Tibétains de manière très inégale. Elle est aussi parsemée d'interdits (où intervient la sanction de l'Etat), qui ne sont pas toujours clairement nommés ou déchiffrables par les Tibétains eux-mêmes. Ces interdits se révèlent tout à coup, lors d'échanges sociaux, économiques ou culturels en apparence anodins, mais qui viennent réveiller les sentiments de frustration, de spoliation et d'injustice étouffés par des années de domination. On pourrait qualifier les escarmouches que j'évoque ici de revendications identitaires, mais on peut aussi se demander si le résultat ne dépasse pas, dans nombre de cas, les intentions de leurs auteurs. D'après mes entretiens avec des réfugiés originaires du Tibet, mais vivant en Inde ou en Occident, la plupart de ces « problèmes politiques » qui ont provoqué un départ précipité en exil étaient de nature réactive, épidermique. Ce n'étaient pas des stratégies mûries de longue date exécutées par un réseau complexe et selon un plan établi. On peut même se demander s'il existe une dissidence au Tibet, sur le modèle des intellectuels d'Amérique latine, de Chine ou de l'ex-URSS. Qu'elles soient le fait de moines formés, d'employés du gouvernement éduqués, ou de paysans analphabètes, ces protestations semblent spontanées, comme une réaction d'outrage que l'on ne pourrait plus contenir. Parmi les réfugiés en Belgique, par exemple, se trouve ce paysan qui était occupé avec ses amis à récolter les précieux champignons médicinaux yartsa gombu ${ }^{47}$, lorsqu'une bande de maraudeurs Chinois est arrivée pour leur faire concurrence. Estimant que le champignon se trouvait sur des terres tibétaines, les villageois ont planté le drapeau tibétain sur le flanc de la colline et tenté de bouter les Chinois dehors. La police est immédiatement intervenue et ceux qui ont été arrêtés ont été lourdement condamnés (à des peines de plus de dix ans de prison).

D’autres récits de réfugiés en Belgique montrent que, parfois, une bagarre dégénère, l'un sort son couteau, l'autre est chinois, et l'altercation est tout de suite interprétée comme un acte d'opposition politique. Le fait d'avoir de la famille en Inde, en prison, ou inversement haut placée dans les services de sûreté joue toujours contre le prévenu. Parfois, le simple fait de se trouver au mauvais endroit au mauvais moment suffit à attirer les soupçons, tel ce moinillon présent par hasard lors de la rébellion de deux moines aînés. Un autre moine, qui répète à un ami des informations sensibles qu'il vient d'apprendre sur un chemin de pèlerinage, est entendu par un tiers et dénoncé. Il n'y a donc pas de critère univoque sur ce qui constitue, en Chine, une activité politique, et ce qui n'en est pas. Ce que l'on sait, ce que l'on ne sait pas, le réseau social, et tout simplement la malchance jouent de concert pour désigner, tout à coup, un individu comme suspect. Et quand il faut fuir, c'est dans l'immédiat, sans préparation, sans réfléchir, «sans se retourner » comme ils le disent souvent. Combien de jeunes hommes n’ont pas laissé leur épouse, combien de mères n'ont pas laissé leur nourrisson, combien d'adolescents n'ont pas laissé derrière eux toute une famille à qui ils n'ont eu le temps de rien dire ? Et lorsqu'ils arrivent en Belgique, les Tibétains ne sont pas égaux de par les blessures qu'ils

47 Cordyceps sinensis. On lui prête des vertus aphrodisiaques et stimulantes. Depuis que des athlètes chinoises lui ont attribué leur succès au début des années quatre-vingt-dix, la popularité et le prix de ce champignon ont prodigieusement grimpé (environ quatre mille euros le kilo sur les marchés chinois), ce qui a hélas provoqué sa cueillette sur-intensive. Il ne pousse que sur le plateau himalayen et constitue dorénavant la rentrée financière principale de nombreux nomades tibétains. 
transportent avec eux. Certains ont dû surmonter de grands abandons, d'autres ont connu des abus en Inde, d'autres encore ont eu le luxe d'avoir le voyage en Occident « payé » par de riches parents.

Par contraste, les récits des exilés comportent des images plus stéréotypées, en particulier sur la police et l'armée. Influencés par les discours hégémoniques de l'exill ${ }^{48}$, par les documentaires occidentaux sur le Tibet, et peut-être aussi par les films hollywoodiens, ils s'attribuent souvent des activités empreintes d'héroïsme et de sentiment nationaliste pantibétain. Il n'y a donc pas que la tradition qui soit inventée à Dharamsala : la modernité, à savoir la manière dont la région est gérée par le gouvernement chinois, est elle aussi inventée. Elle configure l'imaginaire politique des jeunes, et les soude dans des sentiments d'appartenance très forts et très affectifs. Mais, pour nourrir leur ambition de défenseurs de leurs frères opprimés, ces jeunes ont besoin de victimes à soutenir. Or, comme me l'a confié en privé l'un d'entre eux : "Que nous arrivera-t-il lorsqu'il n'y aura plus de prisonniers politiques au Tibet $?{ }^{49}$. Comme l'a proposé Bhabha $(1990: 1)$, tout sens de la nation est issu d'une narrativisation. Les récits des exilés laissent clairement apparaître à quel point ils sont façonnés par les scripts de leur nation. Toutefois, ici comme ailleurs (Bhabha 1990 : 4), cette idée de nation est ambivalente : elle est tout à la fois force de création et de ralliement et imposition de contraintes normatives.

Toutefois, ces considérations sur les projections imaginaires des exilés ne doit pas faire perdre de vue que plusieurs Tibétains de l'exil réfugiés en Belgique sont aussi des activistes politiques qui ont rencontré de « réels » problèmes en Inde. Pour avoir écrit « Free Tibet » sur les murs de l'ambassade de Chine à Delhi, pour avoir chahuté la visite officielle de hauts dirigeants chinois en Inde, et pour s'être rebellés lors de leur détention dans les prisons indiennes, ils sont à présent stigmatisés dans une société qui les tolère mais ne les intègre pas vraiment.

\section{Tibétains de l'exil, Tibétains du Tibet}

Si le but de l'audition au Commissariat est, on l'a vu, de débusquer le mensonge au moyen d'une évaluation de la rationalité narrative du demandeur, l'une de ses visées principales est de départager les Tibétains nés en exil, considérés comme ne relevant pas de la Convention de Genève, et ceux qui sont nés au Tibet. Les premiers ressentent cette distinction comme injuste, car ils savent que les Tibétains nés au Tibet ne mentent pas moins qu'eux (sur leurs prétendues activités politiques comme sur les longues années qu'ils ont passées en Inde), seulement de manière plus habile, et ils les trouvent généralement opportunistes, moins impliqués politiquement (ils se mobilisent effectivement davantage

McGranahan (2005 : 575) vient rappeler à juste titre que les efforts de l'administration en exil pour promouvoir un modèle unifié, toiletté de toute dissension, de la tradition tibétaine et de son combat actuel ont été largement couronnés de succès, mais qu'ils n'ont réussi qu'à " ajourner » (store away for future discussion) les «histoires arrêtées » (arrested histories) de la seconde moitié du vingtième siècle, telles l'importance de la guérilla des Khampas (Tibétains de l'Est) dans le combat politique, version qui pouvait mettre en péril la version offielle. Il faudrait donc se réserver d'estimer les conceptions stéréoptypées des Tibétains de l'exil de manière trop monolithique.

Le Tibetan Centre for Human Rights and Democracy en a dénombré cent seize dans les geôles chinoises en 2006. (http://www.tchrd.org/publications/annual_reports/2006/ar_2006.pdf, p. 17, consulté le 10 mai 2007). 
pour manifester en Belgique, mais il faudrait aussi évaluer la peur des répercussions au pays pour ceux qui sont nés en Chine).

Les examinateurs belges ne recourent pas, comme c'est le cas en Suisse, à des tests linguistiques pour déterminer l'origine des candidats. Leur évaluation porte sur trois types de questions : sur des traces de socialisation minimale en Chine, sur leur connaissance de la géographie régionale, et enfin sur leur mode de vie. A tous les candidats, il est demandé de dessiner le drapeau chinois, la carte d'identité (et de la décrire : comporte-t-elle des inscriptions en tibétain ou seulement en chinois ?) et parfois les billets de banque. Ils doivent énoncer quelques mots en chinois (les candidats ignorent, parfois à leurs dépens, que les examinateurs sont formés dans cette langue), et décrire le système des livrets de résidence (chin. hukou). De nombreuses questions leur sont posées sur les officiers de police (la couleur de leur uniforme, leur dénomination en tibétain ${ }^{50}$ et en chinois). Il leur est aussi demandé de dessiner le plan de leur village, projection en deux dimensions que ceux qui n'ont pas été scolarisés sont incapables de produire. A ceux-ci, on demande alors de placer les diverses maisons, édifices religieux, montagnes et rivières sur trois dimensions, autour d'eux dans la pièce. Tous doivent aussi évaluer les distances entre les villages de leur région (évaluées par temps de marche ou de trajet en bus ${ }^{51}$ ), et nommer les maîtres religieux de leur monastère et des monastères environnants.

Enfin, on les sonde sur leurs compétences d'agriculteurs ou d'éleveurs nomades, selon l'occupation déclarée. Les premiers doivent décrire la perception des taxes agricoles, le système des canaux d'irrigation (la coordination des temps d'irrigation représente effectivement le ciment social d'un village), le rendement d'un are, ou la manière dont les récoltes sont effectuées. Les seconds doivent fournir la terminologie détaillée relative à leurs animaux domestiques, ou décrire les tâches de base des éleveurs ${ }^{52}$. Aux citadins, on demande de décrire la ville et ses aménagements. Les questions destinées aux moines, en revanche, sont moins décisives, puisque la vie quotidienne des religieux est comparable, dans ses grandes lignes, au Tibet et en exil.

Toutes ces questions n'opèrent leur arbitrage que par effet de surprise. Les candidats se sont, au fil des auditions, passés le mot, et ils arrivent dorénavant bien préparés sur ces thèmes, ce qui provoque de nouveaux ajustements dans les questions des examinateurs... D’une certaine manière, ce qui étonne le plus les candidats n'est pas que le Commissariat

50 On trouve parfois, dans la bouche de certains exilés, des formulations naïves telles pulissè (calque de l'anglais police, utilisé en Inde) ou kuten, qui désigne les gardes du corps du Dalai Lama.

51 Ici encore, l'ignorance de certains Tibétains de l'exil est criante. L'un d'entre eux a évalué la distance entre le Potala et le Tashi Lhunpo, les deux palais monastiques les plus importants, à dix minutes à pied, alors qu'ils sont distants de ... trois cent cinquante kilomètres.

52 De grandes variations dialectales affectent la taxinomie des animaux domestiques. Ces termes sont généralement inconnus des paysans sédentaires, et a fortiori des citadins et des exilés. Les catégories tiennent compte du nombre d'années (jusqu'à quatre ou sept ans) et du sexe de l'animal. Ainsi, chez les nomades Abohor du Tibet central, un yak est-il appelé successivement phobe'u, yarpho, shepo et yagu de un à quatre ans, et puis seulement nommé « yak ». La femelle du yak est quant à elle appelée successivement mobe 'u, yarmo, shemo et thünma sur la même période, puis dri. Le principe est le même pour les moutons, les chèvres et les chevaux (informations de Trarong Yangga, Abohor plaisamment échoué à Harvard University). 
cherche à déterminer de quel côté ils sont originaires, ce sont les grands moyens qu'il se donne pour y parvenir. Car eux, les Tibétains, n'ont guère besoin de longues explications pour se catégoriser entre eux. C'est d'ailleurs la première chose qu'ils font lorsqu'ils se rencontrent ${ }^{53}$. Cela ne leur prend qu'une seconde : un regard. Au téléphone également : une phrase. Ils sont bien en mal d'isoler leurs critères. "C'est subtil, c'est un feeling ", dit Tashi. Et leur intuition est infaillible. Visuellement, cela tient à une combinaison des traits morphologiques (plus d'embonpoint en Inde, des pommettes saillantes au Tibet), de la démarche (plus chaloupée en Inde, plus verticale au Tibet), de la façon de se tenir, des choix vestimentaires (plutôt «cool» pour l'Inde, plutôt classique pour le Tibet), du comportement général (plus réservé en Inde), de l'attitude en situation de vis-à-vis (plus de proximité au Tibet) et du thé qu'ils boivent (le chai en Inde, le thé sucré ou salé au Tibet). Dans une foule en Occident, ils se reconnaissent instantanément. Plus exactement, ils reconnaissent d'abord qu'il s'agit d'une personne tibétaine (en dépit de la grande variabilité morphologique des Tibétains), puis de quel type de Tibétain il s'agit. Le cas tibétain, plus exactement la coexistence actuelle de deux manières d'être tibétain, fournit une illustration d'école au concept bourdieusien d'hexis corporelle ${ }^{54}$, « l'habitus fait corps » en quelque sorte. L'histoire collective des Tibétains s'est sédimentée de manière différentielle dans leur corps et dans leurs ressentis, jusqu'à apparaître naturelle, irréfléchie. Le climat, l'alimentation, l'environnement général indien ou chinois, la vie sociale quotidienne, les affects vécus avec le plus de fréquence, les idéologies locales, ont contribué à produire une série de «dispositions durables», à organiser des visions du monde et des grilles de lecture de l' « authenticité » tibétaine, selon deux grand types distincts $^{55}$.

L'enjeu du contentieux le plus douloureux concerne néanmoins l'usage de la langue. Les dialectes et accents régionaux étant si marqués dans la langue tibétaine, l'origine $\mathrm{du}$ locuteur est immédiatement perceptible (un doute subsiste toutefois dans le cas de Tibétains ayant transité longuement dans plusieurs endroits). Les jeunes exilés ont eux aussi leur parler reconnaissable, organisé sur la langue de Lhasa, mais mâtiné de tournures du Tibet occidental. Ce qui cause des frictions importantes entre les Tibétains nés de part et d'autre de la barrière himalayenne réside dans le recours systématique aux termes exogènes que fait dorénavant leur usage de la langue (chinois d'un côté, hindi et anglais de l'autre). Les exilés sont prompts à dénoncer la sinisation du tibétain parlé en Chine mais, confrontés en miroir à une critique équivalente, ils prétendent que hindi et anglais sont des accommodements positifs, voire «branchés ». A la question de l'étiolement de la langue et de la revendication de sa pureté de part et d'autre, il faut encore ajouter l'intonation (une tendance à plus d'assertivité au Tibet), le volume sonore ou la phonétique (une certaine

53 Soulignons qu'en dépit de nombreux cas hybrides, la polarité reste toujours opérante chez les Tibétains. Un réfugié né au Tibet, même s'il a vécu de nombreuses années en Inde, se considérera toujours comme Tibétain du Tibet. Inversement, les nombreux Tibétains de l'exil qui ont passé de longues années à Taiwan et parlent couramment le chinois, se considèrent toujours comme Tibétains d'Inde.

«L'hexis corporelle est la mythologie politique réalisée, incorporée, devenue disposition permanente, manière durable de se tenir, de porter le corps » (Bourdieu 1980 : 117).

55 Il faudrait voir, si l'immigration en Occident produira une troisième hexis corporelle tibétaine. Il faut aussi souligner que la plupart des Tibétains ont transité par plusieurs endroits avant de parvenir en Belgique, et qu'ils se sont nourris d'influences à divers endroits. 
nasalisation au Tibet, une propension à moins articuler en Inde), qui procurent une fiche signalétique immédiate d'un Tibétain. Enfin, les attitudes qui accompagnent la locution sont aussi marquées par des habitudes distinctes (l'écarquillement des yeux pour marquer la désapprobation, les moues, la façon de s'adresser aux autres pour leur demander de l'aide...). Ces différences, pour ténues qu'elles puissent apparaître à un observateur extérieur, préoccupent intensément les Tibétains qui n’ont de cesse de les commenter.

\section{Conclusion}

Comme l'a souligné Malkki (1995: 506), « réfugié » ne désigne ni une catégorie sociale, ni un état ou une subjectivité qui serait commune à tous les réfugiés. C'est un terme qui se réfère plutôt à un processus transitoire, à une négociation entre un avant, un pendant et les espoirs placés dans un après. « Demandeur d'asile » n'est pas davantage une identité, car le statut est par définition temporaire (de deux à quatre ans pour les Tibétains, avant qu'ils ne soient notifiés de leur acceptation éventuelle). Même au sein d'un seul groupe culturel comme les Tibétains, les différences de trajectoires, de bagage et de compétences sont si considérables qu'on serait bien en peine de formuler des généralisations sur leur situation en Belgique. Mais on peut néanmoins esquisser à grands traits la manière dont la plupart d'entre eux ont traversé cette période de tunnel juridique.

Le boom de l'immigration tibétaine en Belgique est particulièrement récent, puisqu'il n'a commencé qu'en 2002. Comme la plupart des néo-réfugiés, les Tibétains ne sont généralement pas au courant des débats (juridiques, politiques, ou d'opinion publique) qui concernent les réfugiés au sein de leur société d'accueil (Dobson 2004 : 47). Ils ne s'identifient ni comme réfugiés (alors qu'ils le faisaient en Inde ou au Népal), ni à d'autres réfugiés, qu'ils ne fréquentent pas. Ils sont parfois hébergés en centres d'accueil fédéraux (ouverts), ou dans des centres d'accueil de la Croix-Rouge, mais ils habitent le plus souvent dans des logements privés, à cinq ou six par chambre, grâce à une aide du CPAS. Les moins éduqués, qui sont aussi les moins curieux, ont l'impression de provenir d'un territoire immense en comparaison de l'Occident, parfois pensé comme un seul pays nommé Angli. Ils sont choqués lorsque leur interlocuteur ne connaît pas le Tibet, ou le Dalai Lama. Jamais associés aux images de réfugiés insécurisants pour la population belge (Gsir et al. 2004 : 263-265), ils sont relativement bien acceptés, dans la mesure où ils sont quasiment invisibles ${ }^{56}$. Pour ceux qui ne parlent ni l'anglais ni l'une des langues officielles en Belgique (issus de villages reculés du Tibet, le plus souvent), les contacts avec la population locale restent purement institutionnels : fréquentation d'un CPAS, d'un médecin, de services d'animation culturelle dans les quartiers, ou d'écoles de devoirs. De ce fait, leur perception de notre mode de vie est généralement tronquée. Le travail qu'ils ont pu trouver en marge de leur couverture sociale, pas ou peu déclaré, relève le plus souvent du secteur agro-alimentaire : le maraîchage, les pépinières, les conserveries de poisson et les abattoirs de poulet en Flandre, et les fruits en Wallonie ${ }^{57}$. D'autres ont obtenu

56 Cet ouvrage propose une enquête comparative sur la diversité des réactions des riverains de six sites d'hébergement de demandeurs d'asile (avec et sans centre d'accueil) en Wallonie et à Bruxelles.

57 L'utilisation des demandeurs d'asile comme main d'œuvre peu onéreuse est généralement le fait d'une population locale elle-même issue de l'immigration (Gsir et al. 2004 : 257-259). «Il y a donc une reproduction de l'exploitation que des immigrés ou leurs descendants ont pu subir» (Id. : 258). 
des petits jobs dans la restauration (chinoise, italienne et belge) ou, mettant à profit leur connaissance des langues (le chinois ou l'anglais), dans l'hôtellerie et les services. Leurs stratégies sont le plus souvent à court terme (amasser de l'argent, payer la dette, renvoyer une somme en Asie), et très rarement à long terme (l'éducation de leurs enfants résidant en Belgique, par exemple). Tant qu'ils ne sont pas fixés sur leur statut, ils rechignent à faire l'effort d'apprendre l'une des langues nationales (ou même l'anglais, pour ceux qui proviennent du Tibet). Leur acceptation en tant que réfugiés politiques décuple leur volonté, et ils maîtrisent alors rapidement les langues dont ils ont besoin. Ils se fréquentent presque exclusivement entre eux ou, plus exactement, forment des petits groupes assez fermés, constitués sur la base d'affinités régionales, de camaraderie scolaire ou d'amitiés nouées en Inde (pour les exilés de longue date comme pour les autres). Leurs rares amis belges sont pour la plupart bouddhistes ou sympathisants de la cause tibétaine.

Aux yeux des Tibétains, le phénomène le plus préoccupant est que, suite à l'afflux des candidats réfugiés depuis 2002, la communauté a de plus en plus de difficultés à maintenir sa cohésion. Une association, la Tibetan community in Belgium ${ }^{58}$, distincte du Bureau du Tibet (l'organe officiel du gouvernement de Dharamsala à Bruxelles) a été fondée de bonne heure pour rassembler ceux qui n'étaient à l'époque qu'une vingtaine. Dirigée par un corps élu, sa fonction principale a été, et reste, l'organisation des grandes fêtes communautaires, telles le nouvel an ou l'anniversaire du Dalai Lama. Aujourd'hui, en dépit d'un nouveau site $w_{e b}{ }^{59}$ qui tente de fédérer les diverses lignes de force tibétaines, les troupes ne répondent plus à l'appel. Des groupes entiers d'Amdowas ou de Khampas préfèrent effectuer des centaines de kilomètres en voiture pour célébrer ces fêtes au sein de leurs réseaux dans les pays limitrophes ou en Suisse. Pourtant, la coordination d'un nombre restreint de compatriotes sur un territoire aussi petit que la Belgique ne devrait pas poser de grands problèmes. Si l'on voulait formuler de manière ironique la raison de cet étiolement (ironique au regard de la situation de la Belgique elle-même), on pourrait dire que les Tibétains qui vivent ici sont obnubilés par leurs propres problèmes communautaires. Certes, les raisons profondes de cette méfiance doivent être recherchées en amont de leur arrivée en Occident, mais la ségrégation qu'ont opérée les instances d'asile belges entre Tibétains d'Inde et Tibétains du Tibet a eu un impact puissant sur les relations intra-communautaires. En dévalorisant les premiers, en octroyant plus facilement à d'autres des droits sociaux confortables (un statut reconnu et valorisé, le droit de travailler et surtout le regroupement familial, véritable dopant de la santé mentale des réfugiés), cette ségrégation a laissé de profondes séquelles, à la fois collectives et individuelles. Volontiers fanfarons à leur arrivée (parlant couramment l'anglais, sapés de manière branchée, et souvent plus à l'aise avec les Belges), les Tibétains de l'exil ont vu tout à coup le ciel se refermer au-dessus de leur tête. Une rumeur persistante qui circule parmi les Tibétains de France affirme que les Tibétains de Belgique sont en train de devenir « fous » (entendons, dépressifs ${ }^{60}$ ). Via son site web, la communauté tibétaine de

En anglais dans l'intitulé. Suisse dans les années soixante, dans des situations extrêmes d'anomie (Korom 1999, pp. 9-10). 
Belgique a envoyé un formulaire de sondage à ses membres, qui se terminait par ces mots : «How do you survive these days ? " ${ }^{61}$. L'échec en procédure d'asile est souvent vécu subjectivement par les exilés comme un échec personnel en tibétanité, une impuissance à mettre en œuvre le projet dans lequel ils ont été socialisés depuis l'enfance. Quelle est dès lors leur valeur? Qu'ont-ils à apporter au monde? Et pourquoi ces autres Tibétains qui n'ont pas fait d'études et qui n'ont, à ce qu'il leur semble, aucune conscience politique, méritent-ils les honneurs du simple fait de leur naissance?

A tant se préoccuper de la définition de leur tibétanité et de l'acculturation chinoise ou indienne de la partie adverse, ces réfugiés en sont venus à s'aveugler, me semble-t-il, sur l'acculturation qui les menace personnellement au plus haut point : l'acculturation occidentale, dans sa version belge. Ceux qui résident en Belgique actuellement ne sont que les têtes de pont. Sans recul, il est difficile d'estimer le degré de persistance culturelle et l'impact différentiel des modèles d'intégration des divers pays européens. A partir d'un pool de migrants restreint, de Tapia (2001 : 11) avait conclu à une intégration sans heurts des Tibétains en France, facilitée par une forte cohésion sociale et une option rapidement marquée pour l'intégration (Id. : 11-12). La situation en Belgique sera certainement pertinente à étudier dans peu de temps, étant donné le grand nombre de réfugiés et la coexistence d'une cohésion sociale forte dans l'environnement immédiat des candidats, et de tensions importantes dans la communauté dans son ensemble.

On dispose de plus de recul aux Etats-Unis, puisque l'immigration y a commencé dix ans auparavant. La seconde génération, celle des Tibétains qui y sont nés, montre déjà des signes de désintérêt pour les traditions et veut s'intégrer à la culture américaine (Korom 1999, pp. 15-16). Par comparaison avec les études publiées, il apparaît que l'expression de la tibétanité lors des grands rassemblements est bien moins normative en Belgique. Yeh (2006, p. 825) évoque les sanctions qui frappent les jeunes Tibétains qui pratiquent des traditions culturelles non authentiques, telles le rap ( $«$ authorized articulation of only one Tibetan identity »). Par ailleurs, si l'on doit croire l'avis de Hess (2006) sur l'efficacité du «nationalisme transnational» des Tibétains résidant en Amérique, la naturalisation américaine étant conçue comme un levier politique, que penser dès lors de l'adoption de la citoyenneté belge par des réfugiés qui ignoraient jusqu'à l'existence de ce pays le jour de leur départ? La Belgique est-elle pour eux, comme pour tous ceux qui n'auront pas été reconnus, une terre d'arrivée, ou une planche pour un nouveau départ ? de Tapia (2001 : 2) avait déjà été surpris par le fait que la question de l'intention de leur migration vers l'Ouest restait sans réponse, à l'opposé d'une théorie rationnelle de la migration qui inonde les médias et selon laquelle un individu pèse le coût de sa vie dans son pays d'origine, et le compare au coût humain de la migration en Europe - dans les cas où la migration est volontaire, bien sûr.

Les Tibétains de Belgique se réinventent et se chamaillent en permanence : quel est le contenu de leur tradition? En quoi la modernité qu'ils se choisissent leur permetelle de conserver leur tibétanité ? Et avec quels fils de la tradition tisser des solutions modernes aux défis qui se posent à eux ? Si, ainsi que le suggérait Yonten en exergue de cet article, la tradition de la modernité est de nature migratoire, la question de l'ancrage

61 http://tibetancommunity.be/ (accédé le 10 juin 2007. Le questionnaire n'est plus en ligne). 
dans un lieu où l'on se sent en définitive chez soi (home, homeland) ne manquera pas de refaire surface. Ils se rêvent descendants d'orgueilleux nomades qui arpentaient les hauts plateaux enneigés ${ }^{62}$. Les voilà nomades urbains, incertains de leur avenir. Une fois cette première phase de transition dépassée, une longue suite reste à écrire sur le type de sédentarité que ces montagnards auront choisie pour s'adapter au plat pays.

62 L'image est belle, et parfois mobilisée par les Tibétains. Mais c'est en fait une contre-vérité, car la paysannerie sédentaire semble avoir précédé l'implantation de la culture nomade. 


\section{Références bibliographiques}

AsSAYAG, Jackie, 1989. « Bouddha en exil : La grande transformation des réfugiés tibétains dans le sud de l'Inde (Mundgod-Karnataka) », Archives européennes de sociologie, 30 (1), pp. 63-89.

BARNETT, Robert,

2001. "'Violated Specialness': Western Political Representations of Tibet », dans Thierry Dodin et Heinz Räther (éds.), Imagining Tibet: Perceptions, Projections, and Fantasies, pp. 269-316. Boston, Wisdom Publications.

2006. « Beyond the Collaborator-Martyr Model: Strategies of Compliance, Opportunism, and Opposition within Tibet », dans Barry Sautmann et June Teufel Dreyer (éds.), Contemporary Tibet: Politics, Development, and Society in a Disputed Region, pp. 25-66. Armonk : M.E. Sharpe.

Внавна, Homi, 1990. «Introduction: Narrating the Nation », dans Homi Bhabha (éd.), Nation and Narration, pp. 1-7. London, New York, Routledge.

Bourdieu, Pierre, 1980. Le Sens pratique. Paris, Editions de Minuit.

Buffetrille, Katia et Charles Ramble, 1998. Tibétains, 1959-1999: Quarante ans de colonisation. Paris, Autrement.

Buffetrille, Katia et Anne-Marie Blondeau, 2002. Le Tibet est-il chinois ? Paris: Albin Michel.

Crescenzi, Antonella et allii, 2002. « Effect of Political Imprisonment and Trauma History on Recent Tibetan Refugees in India », Journal of Traumatic Stress, 15 (5), pp. 369-375.

DE TAPIA, Stéphane, 2001. « Des steppes de Haute Asie à l'Ile-de-France : Kazakhs, Kalmouks et Tibétains », Migration Etudes, Synthèse de travaux sur l'immigration et la présence étrangère en France, 98, pp. 1-16.

Dobson, Stephen, 2004. Cultures of Exile and the Experience of Refugeeness. Bern / New York, P. Lang.

Ginet, Pierre-Yves, 2000. Tibet en exil : entre mythes et réalités. Villeurbanne, Golias.

Garratt, Kevin, 1997. «Tibetan Refugees, Asylum Seekers, Returnees and the Refugees Convention Predicaments, Problems and Prospects », Tibet Journal 22 (3), pp. 18-56.

Gsir Sonia, Fabienne Scandella, Marco Martiniello, et Andrea Rea, 2004. Les Belges francophones face aux demandeurs d'asile. Gand, Academia Press (Série : Problèmes actuels concernant la cohésion sociale).

Granhag, Pär Anders, LeifA. Strömwall, et Maria Hartwig, 2005. « Granting Asylum or not ? Migration Board Personnel's Beliefs about Deception », Journal of Ethnic and Migration Studies, 31 (1), pp. 29-50.

Good, Anthony, 2004. " 'Undoubtedly an Expert ?' Anthropologists in British Asylum Courts », Journal of the Royal Anthropological Institute, 10, pp. 113-133.

Havinga, Tetty et Anita Böcker, 1999. «Country of Asylum by Choice or by Chance: Asylum Seekers in Belgium, the Netherlands and the U.K. », Journal of Ethnic and Migration Studies, 25 (1), pp. 43-61.

HerzFeld, Michael, 2005. Cultural Intimacy : Social Poetics in the Nation-State. New York, Routledge (seconde edition).

Hesford, Wendy S., 2004. « Documenting Violations: Rhetorical Witnessing and the Spectacle of Distant Suffering », Biography, 27 (1), pp. 104-144.

Hess, Julia M., 2006. «Statelessness and the State: Tibetans, Citizenship, and Nationalist Activism in a Transnational World », International Migration, 44 (1), pp. 79-103.

Houston, Seth et Richard Wright, 2003. «Making and Remaking TibetanDiasporic Identities », Social \& Cultural Geography, 4 (2), pp. 217-232.

Korom, Frank J., 1999. « Tibetans in Exile: A Euro-American Perspective », Passages: Journal of Transnational and Transcultural Studies, 1 (1), pp. 1-23. 
Lopez, Donald S., 2003 [1998]. Fascination tibétaine. Du bouddhisme, de l'Occident et de quelques mythes. Paris, Autrement.

MALKKI, Liisa,

1992. « National Geographic: The Rooting of Peoples and the Territorialization of National Identity among Scholars and Refugees », Cultural Anthropology, 7 (1), pp. 24-44.

1995. « Refugees and Exile: From 'Refugee Studies' to the National Order of Things », Annual Review of Anthropology, 24, pp. 495-523.

McGranahan, Carole, 2005, «Truth, Fear, and Lies: Exile Politics and Arrested Histories of the Tibetan Resistance », Cultural Anthropology, 20, 4, pp. 570-600.

McLagan, Meg, 2002. "Spectacles of Difference: Cultural Activism and the Mass Mediation of Tibet », dans Faye Ginsburg, Lila Abu-Lughod et Brian Larkin (éds.), Media Worlds: Anthropology on New Terrain, pp. 90-111. Berkeley, University of California Press.

NowAK, Margaret, 1984. Tibetan Refugees: Youth and the New Generation of Meaning. New Brunswick, Rutgers University Press.

PäLdÈn Gyatso, 1997. Le Feu sous la neige. Mémoires d'un moine tibétain. Arles, Actes Sud.

Shakya, Tsering, 1999. The Dragon in the Land of Snows: a History of Modern Tibet since 1947. Londres, Pimlico.

SPerling, Elliot, 2004. The Tibet-China Conflict: History and Polemics. Washington, East-West Center.

TiN (Tibet Information Network), 2002. « Decline in Refugee Numbers as China and Nepal Tighten Security on Border », TIN news update, 2 janvier.

VAlluy, Jérôme, 2005. « Vrai ou faux réfugiés ? », Espaces Temps (Réfléchir les sciences sociales) - Les Cahiers 89-90, pp. 96-103.

YEH, Emily,

2002. «Will the Real Tibetan Please Stand up ?: Identity Politics in the Tibetan Diaspora », dans Christiaan P. KLIEGER (éd.), Tibet, Self, and the Tibetan Diaspora: Voices of Difference. Proceedings of the $9^{\text {th }}$ Seminar of the International Association for Tibetan Studies, Leiden, Brill, pp. 229-254.

2007. «Exile meets Homeland: Politics, Performance and Authenticity in the Tibetan Diaspora », Environment \& Planning D: Society and Space 25 (4), pp. 648-667.

YeH, Emily T. et Kunga T. Lama, 2006. « Hip-hop Gangsta or most Deserving of Victims ?: Transnational Migrant Identities and the Paradox of Tibetan Racialization in the USA », Environment \& Planning A, 38, pp. 809-829. 